\title{
A Backward Analysis for Constraint Logic Programs
}

\author{
ANDY KING \\ University of Kent at Canterbury, Canterbury, CT2 7NF, UK. \\ email: amk@ukc.ac.uk
}

LUNJIN LU

Oakland University, Rochester, MI 48309, USA.

email: l2lu@oakland.edu

\begin{abstract}
One recurring problem in program development is that of understanding how to re-use code developed by a third party. In the context of (constraint) logic programming, part of this problem reduces to figuring out how to query a program. If the logic program does not come with any documentation, then the programmer is forced to either experiment with queries in an ad hoc fashion or trace the control-flow of the program (backward) to infer the modes in which a predicate must be called so as to avoid an instantiation error. This paper presents an abstract interpretation scheme that automates the latter technique. The analysis presented in this paper can infer moding properties which if satisfied by the initial query, come with the guarantee that the program and query can never generate any moding or instantiation errors. Other applications of the analysis are discussed. The paper explains how abstract domains with certain computational properties (they condense) can be used to trace control-flow backward (right-to-left) to infer useful properties of initial queries. A correctness argument is presented and an implementation is reported.
\end{abstract}

\section{Introduction}

The myth of the lonely logic programmer writing a program in isolation is just that: a myth. Applications (and application components) are usually implemented and maintained by a team. One consequence of this is a significant proportion of the program development effort is devoted to understanding code developed by another. One advantage of (constraint) logic programs for software development is that their declarative nature makes them less opaque than, say, $\mathrm{C}++$ programs. One disadvantage of logic programs over $\mathrm{C}++$ programs, however, is that the signature (argument types) of a predicate do not completely specify how the predicate should be invoked. In particular, a call to a predicate from an unexpected context may generate an error if an argument of the call is insufficiently instantiated (even if the program and query are well-typed). This is because logic programs contain builtins and calls to these builtins often impose moding requirements on the query. If the program is developed by another programmer, it may not be clear how to 
query a predicate so as to avoid an instantiation error. In these circumstances, the programmer will often resort to a trial and error tactic in their search for an initial call mode. This can be both frustrating and tedious and, of course, cannot guarantee coverage of all the program execution paths. This paper presents an analysis for inferring moding properties which, if satisfied by the initial query, ensure that the program does not generate instantiation errors. Of course, it does not mean that the inferred call has the form exactly intended by the original programmer - no analysis can do that - the analysis just recovers mode information. Nevertheless, this is a useful first step in understanding the code developed by another.

The problem of inferring initial queries which do not lead to instantiation errors is an instance of the more general problem of deducing how to call a program so that it conforms to some desired property, for example, calls to builtins do not error, the program terminates, or calls to builtins behave predictably. The backward analysis presented in this paper is designed to infer conditions on the query which, if satisfied, guarantee that resulting derivations satisfy a property such as one of those above. Specifically, the analysis framework can be instantiated to solve the following analysis problems:

- Builtins and library functions can behave unpredictably when called with infinite rational trees. For example, the query ?- $\mathrm{X}=\mathrm{X}+\mathrm{X}, \mathrm{Y}$ is $\mathrm{X}$ will not terminate in SICStus Prolog because the arithmetic operator expects its input to be a finite tree rather than an infinite rational tree. Moreover, the standard term ordering of Prolog does not lift to rational trees, so the builtin sort can behave unpredictably when sorting rational trees. These problems (and related problems with builtins) motivate the use of dependency analysis for tracking which terms are definitely finite (Bagnara et al., 2001). The basic idea is to describe the constraint $x=f\left(x_{1}, \ldots, x_{n}\right)$ by the Boolean function $x \Leftrightarrow \wedge_{i=1}^{n} x_{i}$ which encodes that $x$ is bound to a finite tree iff each $x_{i}$ is bound to a finite tree. Although not proposed in the context of backward analysis (Bagnara et al., 2001), the framework proposed in this paper can be instantiated with a finite tree dependency domain to infer finiteness properties on the query which, if satisfied, guarantee that builtins are not called with problematic arguments.

- Termination inference is the problem of inferring initial modes for a query that, if satisfied, ensure that a logic program terminates. This problem generalises termination checking which verifies program termination for a class of queries specified by a given mode. Termination inference dates back to (Mesnard, 1996) but it has been recently observed (Genaim \& Codish, 2001) that the missing link between termination checking and termination inference is backward analysis. A termination inference analyser is reported in (Genaim \& Codish, 2001) composed from two components: a standard termination checker (Codish \& Taboch, 1999) and the backward analysis described in this paper. The resulting analyser is similar to the cTI analyser of (Mesnard \& Neumerkel, 2001) - the main difference is its design as two existing black- 
box components which, according to (Genaim \& Codish, 2001), simplifies the formal justification and implementation.

- Mode analysis is useful for implementing ccp programs. In particular (Debray et al., 1992) explains how various low-level optimisations, such as returning output values in registers, can be applied if goals can be scheduled left-toright without suspension. If the guards of the predicates are re-interpreted as moding requirements, then the backward mode analysis can infer sufficient conditions for avoiding deadlock under left-to-right scheduling. The analysis presented in this paper thus has applications outside program development.

To summarise, the analysis presented in this paper can deduce properties of the call which, if satisfied, guarantee that resulting derivations fulfill some desired property. The analysis is unusual in that it applies lower approximation (see 2.4.1) as well as upper approximation (see 2.3.1); it is formulated in terms of a greatest fixpoint calculation (see 2.4) as well as least fixpoint calculation (see 2.3); the analysis also imposes some unusual restrictions on the abstract domain (see 2.4.6).

\subsection{Backward analysis}

Backward analysis has been applied extensively in functional programming in, among other things, projection analysis (Wadler \& Hughes, 1987), stream strictness analysis (Hall \& Wise, 1989), inverse image analysis (Dyber, 1991), etc. By reasoning about the context of a function application, these analyses can identify opportunities for eager evaluation that are missed by (forward) strictness analysis as proposed by (Mycroft, 1981). Furthermore, backward reasoning on imperative programs dates back to the early days of static analysis (Cousot \& Cousot, 1982). By way of contrast, backward analysis has been rarely applied in logic programming. One notable exception is the demand analysis of (Debray, 1993). This analysis infers the degree of instantiation necessary for the guards of a concurrent constraint program (ccp) to reduce. It is a local analysis that does not consider the possible suspension of body calls. This analysis detects those (uni-modal) predicates which can be implemented with specialised suspension machinery. A more elaborate backward analysis for ccp is presented by (Falaschi et al., 2000). This demand analysis infers how much input is necessary for a procedure to generate a certain amount of output. This information is useful for adding synchronisation (ask) constraints to a procedure to delay execution and thereby increase grain size, and yet not introduce deadlock. (Section 7 provides more extensive and reflective review of the related work.)

\subsection{Contributions}

Our work is quite different. As far as we are aware, it is unique in that it focuses on the backward analysis of (constraint) logic programs with left-to-right scheduling. Specifically, our work makes the following practical and theoretical contributions:

- it shows how to compute an initial mode of a predicate which is safe in that 
if a query is at least as instantiated as the inferred mode, the execution is guaranteed to be free from instantiation errors. The modes inferred are often disjunctive, sometimes surprising and, for the small predicates that we verified by hand, appear to be optimal.

- it specifies a practical algorithm for calculating initial modes that is straightforward to implement in that it reduces to two bottom-up fixpoint calculations. Furthermore, this backward analysis problem cannot be solved with any existing abstract interpretation machinery.

- to the best our knowledge, it is the first time domains that are closed under Heyting completion (Giacobazzi \& Scozzari, 1998), or equivalently are condensing (Marriott \& Søndergaard, 1993), have been applied to backward analysis. Put another way, our work adds credence to the belief that condensation is an important property in the analysis of logic programs.

The final point requires some unpacking. Condensation was originally proposed in (Langen, 1991), though arguably the simplest statement of this property (Marriott \& Søndergaard, 1993) is for downward closed domains such as Pos (Armstrong et al., 1998) and the Pos-like type dependency domains (Codish \& Lagoon, 2000). Suppose that $f: X \rightarrow X$ is an abstract operation on a downward closed domain $X$ equipped with an operation $\wedge$ that mimics unification or constraint solving. $X$ is condensing iff $x \wedge f(y)=f(x \wedge y)$ for all $x, y \in X$. Hence, if $X$ is condensing, $x \wedge f($ true $)=f(x)$ where true represents the weakest abstract constraint. More exactly, if $f($ true) represents the result of the goal-independent analysis, and $f(x)$ the result of the goal-dependent one with an initial constraint $x$, then the equivalence $f(x)=x \wedge f($ true) enables goal-dependent analysis to be performed in a goal-independent way without loss of precision. This, in turn, can simplify the implementation of an analyser (Armstrong et al., 1998). Because of this, domain refinement machinery has been devised to enrich a domain with new elements to obtain the desired condensing property (Giacobazzi \& Scozzari, 1998). It turns out that it is always possible to systematically design a condensing domain for a

given downward closed property (Giacobazzi \& Scozzari, 1998)[Theorem 8.2] by applying Heyting completion. Conversely, under some reasonable hypotheses, all condensing domains can be reconstructed by Heyting completion (Giacobazzi \& Scozzari, 1998)[Theorem 8.3]. One consequence of this is that condensing domains come equipped with a (pseudo-complement) operator and this turns out to be an operation that is important in backward analysis. To summarise, machinery has been developed to synthesise condensing domains and condensing domains provide operations suitable for backward analysis.

\subsection{Organisation of the paper}

The rest of the paper is structured as follows. Section 2 introduces the key ideas of the paper in an informal way through a worked example. Section 3 introduces the necessary preliminaries for the formal sections that follow. Section 4 presents an operational semantics for constraint logic programs with assertions in which the set of program states is augmented by a special error state. Section 5 develops a 
semantics which computes those initial states that cannot lead to the error state. The semantics defines a framework for backward analysis and formally argues correctness. Section 6 describes an instantiation of the framework for mode analysis. Section 7 reviews the related work and section 8 concludes. Much of the formal machinery is borrowed directly from (Giacobazzi et al., 1995; Giacobazzi \& Scozzari, 1998) and in particular the reader is referred to (Giacobazzi et al., 1995) for proofs of the semantic results stated in section 3 (albeit presented in a slightly different form). To aid continuity in the paper, the remaining proofs are relegated to appendix A.

\section{Worked example}

\subsection{Basic components}

This section informally presents an abstract interpretation scheme which infers how to query a given predicate so as to avoid run-time moding errors. In other words, the analysis deduces moding properties of the call that, if satisfied, guarantee that resulting derivations cannot encounter an instantiation error. To illustrate, consider the Quicksort program listed in the left column of figure 1. This is the first ingredient of the analysis: the input program. The second ingredient is an abstract domain which, in this case, is Pos. Pos is the domain of positive Boolean functions, that is, the set of functions $f:\{0,1\}^{n} \rightarrow\{0,1\}$ such that $f(1, \ldots, 1)=1$. Hence $x \vee y \in$ Pos since $1 \vee 1=1$ but $\neg x \notin$ Pos since $\neg 1=0$. Pos is augmented with the bottom element 0 with 1 being the top element. The domain is ordered by entailment $\models$ and, in this example, will be used to represent grounding dependencies.

Pos comes equipped with the logical operations: conjunction $\wedge$, disjunction $\vee$, implication $\Rightarrow$ (and thus bi-implication $\Leftrightarrow$ ). Conjunction is used to conjoin the information from different body atoms, while disjunction is used to combine the information from different clauses. Conjunction and disjunction, in turn, enable two projection operators to be defined: $\exists_{x}(f)=f[x \mapsto 0] \vee f[x \mapsto 1]$ and $\forall_{x}(f)=f^{\prime}$ if $f^{\prime} \in$ Pos otherwise $\forall_{x}(f)=0$ where $f^{\prime}=f[x \mapsto 0] \wedge f[x \mapsto 1]$. Note that although $f[x \mapsto 0] \vee f[x \mapsto 1] \in$ Pos for all $f \in$ Pos it does not follow that $f[x \mapsto 0] \wedge f[x \mapsto$ 1] $\in$ Pos for all $f \in$ Pos. Indeed, $(x \Leftarrow y)[x \mapsto 0] \wedge(x \Leftarrow y)[x \mapsto 1]=\neg y$. Both operators are used to project out the body variables that are not in the head of a clause. Specifically, these operators eliminate the variable $x$ from the formula $f$. They are dual in the sense that $\forall_{x}(f) \models f \models \exists_{x}(f)$. These are the basic components of the analysis.

\subsection{Normalisation and abstraction}

The analysis components are assembled in two steps. The first is a bottom-up analysis for success patterns, that is, a bottom-up analysis which infers the groundness dependencies which are known to be created by each predicate regardless of the calling pattern. This step is a least fixpoint (lfp) calculation. The second step is a bottom-up analysis for input modes (the objective of the analysis). This step is a 


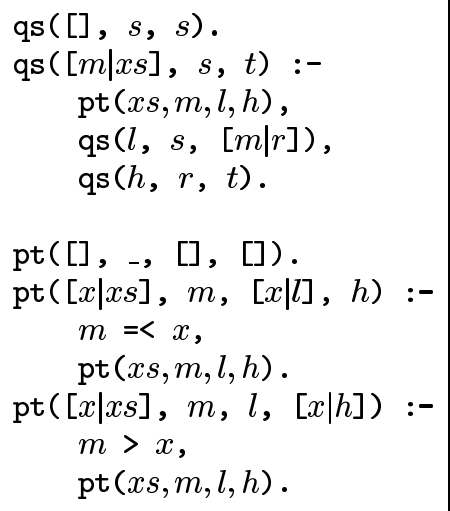

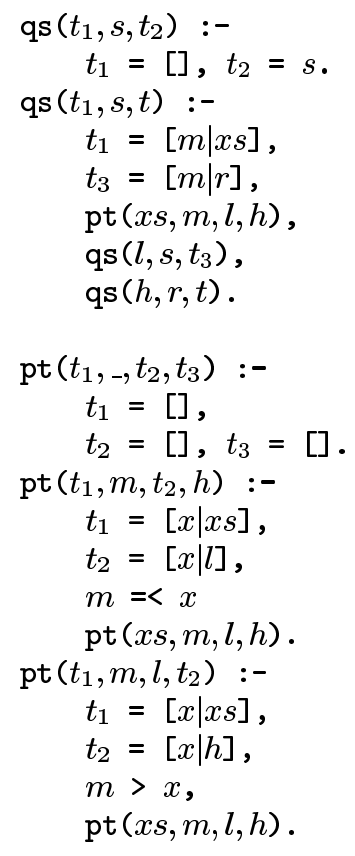

Fig. 1. Quicksort: raw, normalised and abstracted

greatest fixpoint (gfp) computation. To simplify both steps, the program is put into a form in which the arguments of head and body atoms are distinct variables. This gives the normalised program listed in the centre column of figure 1. This program is then abstracted by replacing each Herbrand constraint $x=f\left(x_{1}, \ldots, x_{n}\right)$ with a formula $x \Leftrightarrow \wedge_{i=1}^{n} x_{i}$ that describes its grounding dependency. This gives the abstract program listed in the right column of figure 1 . The formula 1 in the assertion represents true whereas the formulae $g_{i}$ that appear in the abstract program are as follows:

$$
\begin{aligned}
& g_{1}=t_{1} \wedge\left(t_{2} \Leftrightarrow s\right) \\
& g_{4}=t_{1} \Leftrightarrow(x \wedge x s) \wedge t_{2} \Leftrightarrow(x \wedge l) \\
& g_{2}=t_{1} \Leftrightarrow(m \wedge x s) \wedge t_{3} \Leftrightarrow(m \wedge r) \quad g_{5}=t_{1} \Leftrightarrow(x \wedge x s) \wedge t_{2} \Leftrightarrow(x \wedge h) \\
& g_{3}=t_{1} \wedge t_{2} \wedge t_{3} \\
& g_{6}=m \wedge x
\end{aligned}
$$

Builtins that occur in the source, such as the tests $=<$ and $>$, are handled by augmenting the abstract program with fresh predicates, $=<^{\prime}$ and $>^{\prime}$, which express the grounding behaviour of the builtins. The $\diamond$ symbol separates an assertion (the required mode) from another Pos formula describing the grounding behaviour of a successful call to the builtin (the success mode). For example, the formula $g_{6}$ left of $\diamond$ in the $=<^{\prime}$ clause asserts that the $=<$ test will error if its first two arguments are not ground, whereas the $g_{6}$ right of $\diamond$ describes the state that holds if the test succeeds. These formulae do not coincide for all builtins (see Table 1). For quicksort, the only non-trivial assertions arise from builtins. This would change if the programmer introduced assertions for verification (Puebla et al., 2000a). 


\subsection{Least fixpoint calculation}

An iterative algorithm is used to compute the lfp and thereby characterise the success patterns of the program. A success pattern is a pair consisting of an atom with distinct variables for arguments paired with a Pos formula over those variables. Renaming and equality of formulae induce an equivalence between success patterns which is needed to detect the fixpoint. The patterns $\langle p(u, w, v), u \wedge(w \Leftrightarrow v)\rangle$ and $\left\langle p\left(x_{1}, x_{2}, x_{3}\right),\left(x_{3} \Leftrightarrow x_{2}\right) \wedge x_{1}\right\rangle$, for example, are considered to be identical: both express the same inter-argument groundness dependencies. Each iteration produces a set of success patterns: at most one pair for each predicate in the program.

\subsubsection{Upper approximation of success patterns}

A success pattern records an inter-argument groundness dependency that describes the binding effects of executing a predicate. If $\langle p(\vec{x}), f\rangle$ correctly describes the predicate $p$, and $g$ holds whenever $f$ holds, then $\langle p(\vec{x}), g\rangle$ also correctly describes $p$. Success patterns can thus be approximated from above without compromising correctness.

Iteration is performed in a bottom-up fashion and commences with $F_{0}=\emptyset . F_{j+1}$ is computed from $F_{j}$ by considering each clause $p(\vec{x}) \leftarrow d \diamond f, p_{1}\left(\vec{x}_{1}\right), \ldots, p_{n}\left(\vec{x}_{n}\right)$ in turn. Initially $F_{j+1}=\emptyset$. The success pattern formulae $f_{i}$ for the $n$ body atoms are conjoined with $f$ to obtain $g=f \wedge \wedge_{i=1}^{n} f_{i}$. Variables not present in $p(\vec{x}), Y$ say, are then eliminated from $g$ by computing $g^{\prime}=\exists_{Y}(g)$ (weakening $g$ ) where $\exists_{\left\{y_{1} \ldots y_{n}\right\}}(g)=\exists_{y_{1}}\left(\ldots \exists_{y_{n}}(g)\right)$. Weakening $g$ does not compromise correctness because success patterns can be safety approximated from above.

\subsubsection{Weakening upper approximations}

If $F_{j+1}$ already contains a pattern of the form $\left\langle p(\vec{x}), g^{\prime \prime}\right\rangle$, then this pattern is replaced with $\left\langle p(\vec{x}), g^{\prime} \vee g^{\prime \prime}\right\rangle$, otherwise $F_{j+1}$ is revised to include $\left\langle p(\vec{x}), g^{\prime}\right\rangle$. Thus the success patterns become progressively weaker on each iteration. Again, correctness is preserved because success patterns can be safety approximated from above.

\subsubsection{Least fixpoint calculation for Quicksort}

For brevity, let $\vec{u}=\left\langle x_{1}, x_{2}\right\rangle, \vec{v}=\left\langle x_{1}, x_{2}, x_{3}\right\rangle$ and $\vec{w}=\left\langle x_{1}, x_{2}, x_{3}, x_{4}\right\rangle$. Then the lfp for the abstracted Quicksort program is obtained (and checked) in the following 3 iterations:

$$
F_{1}=\left\{\begin{array}{c}
\left\langle\mathrm{qs}(\vec{v}), x_{1} \wedge\left(x_{2} \Leftrightarrow x_{3}\right)\right\rangle \\
\left\langle\mathrm{pt}(\vec{w}), x_{1} \wedge x_{3} \wedge x_{4}\right\rangle \\
\left\langle=<^{\prime}(\vec{u}), x_{1} \wedge x_{2}\right\rangle \\
\left.\langle\rangle^{\prime}(\vec{u}), x_{1} \wedge x_{2}\right\rangle
\end{array}\right\} \quad F_{2}=\left\{\begin{array}{c}
\left\langle\mathrm{qs}(\vec{v}), x_{2} \Leftrightarrow\left(x_{1} \wedge x_{3}\right)\right\rangle \\
\left\langle\mathrm{pt}(\vec{w}), x_{1} \wedge x_{3} \wedge x_{4}\right\rangle \\
\left\langle=<^{\prime}(\vec{u}), x_{1} \wedge x_{2}\right\rangle \\
\left\langle>^{\prime}(\vec{u}), x_{1} \wedge x_{2}\right\rangle
\end{array}\right\}
$$

Finally, $F_{3}=F_{2}$. The space of success patterns forms a complete lattice which ensures that a lfp (a most precision solution) exists. The iterative process will always terminate since the space is finite and hence the number of times each 
success pattern can be updated is also finite. Moreover, it will converge onto the lfp since iteration commences with the bottom element $F_{0}=\emptyset$.

Observe that $F_{2}$, the lfp, faithfully describes the grounding behaviour of quicksort: a qs goal will ground its second argument if it is called with its first and third arguments already ground and vice versa. Note that assertions are not considered in the lfp calculation.

\subsection{Greatest fixpoint calculation}

A bottom-up strategy is used to compute a gfp and thereby characterise the safe call patterns of the program. A safe call pattern describes queries that do not violate the assertions. A call pattern has the same form as a success pattern (so there is one call pattern per predicate rather than one per clause). One starts with assuming no call causes an error and then checks this assumption by reasoning backwards over all clauses. If an assertion is violated, the set of safe call patterns for the involved predicate is strengthened (made smaller), and the whole process is repeated until the assumptions turn out to be valid (the gfp is reached).

\subsubsection{Lower approximation of safe call patterns}

Iteration commences with $D_{0}=\{\langle p(\vec{x}), 1\rangle \mid p \in \Pi\}$ where $\Pi$ is the set of predicate symbols occurring in the program. An iterative algorithm incrementally strengthens the call pattern formulae until they only describe queries which lead to computations that satisfy the assertions. Note that call patterns describe a subset (rather than a superset) of those queries which are safe. Call patterns are thus lower approximations in contrast to success patterns which are upper approximations. Put another way, if $\langle p(\vec{x}), g\rangle$ correctly describes some safe call patterns of $p$, and $g$ holds whenever $f$ holds, then $\langle p(\vec{x}), f\rangle$ also correctly describes some safe call patterns of $p$. Call patterns can thus be approximated from below without compromising correctness (but not from above).

$D_{k+1}$ is computed from $D_{k}$ by considering each $p(\vec{x}) \leftarrow d \diamond f, p_{1}\left(\vec{x}_{1}\right), \ldots, p_{n}\left(\vec{x}_{n}\right)$ in turn and calculating a formula that characterises its safe calling modes. Initially set $D_{k+1}=D_{k}$. A safe calling mode is calculated by propagating moding requirements right-to-left by repeated application of the logical operator $\Rightarrow$. More exactly, let $f_{i}$ denote the success pattern formula for $p_{i}\left(\vec{x}_{i}\right)$ in the previously computed lfp and let $d_{i}$ denote the call pattern formula for $p_{i}\left(\vec{x}_{i}\right)$ in $D_{k}$. Set $e_{n+1}=1$ and then compute $e_{i}=d_{i} \wedge\left(f_{i} \Rightarrow e_{i+1}\right)$ for $1 \leq i \leq n$. Each $e_{i}$ describes a safe calling mode for the compound goal $p_{i}\left(\vec{x}_{i}\right), \ldots, p_{n}\left(\vec{x}_{n}\right)$.

\subsubsection{Intuition and explanation}

The intuition behind the symbolism is that $d_{i}$ represents the demand that is already known for $p_{i}\left(\vec{x}_{i}\right)$ not to error whereas $e_{i}$ is $d_{i}$ possibly strengthened with extra demand so as to ensure that the sub-goal $p_{i+1}\left(\vec{x}_{i+1}\right), \ldots, p_{n}\left(\vec{x}_{n}\right)$ also does not error when executed immediately after $p_{i}\left(\vec{x}_{i}\right)$. Put another way, anything larger than $d_{i}$ 
may possibly cause an error when executing $p_{i}\left(\vec{x}_{i}\right)$ and anything larger than $e_{i}$ may possibly cause an error when executing $p_{i}\left(\vec{x}_{i}\right), \ldots, p_{n}\left(\vec{x}_{n}\right)$.

The basic inductive step in the analysis is to compute an $e_{i}$ which ensures that $p_{i}\left(\vec{x}_{i}\right), \ldots, p_{n}\left(\vec{x}_{n}\right)$ does not error, given $d_{i}$ and $e_{i+1}$ which respectively ensure that $p_{i}\left(\vec{x}_{i}\right)$ and $p_{i+1}\left(\vec{x}_{i+1}\right), \ldots, p_{n}\left(\vec{x}_{n}\right)$ do not error. This step translates a demand after the call to $p_{i}\left(\vec{x}_{i}\right)$ into a demand before the call to $p_{i}\left(\vec{x}_{i}\right)$. The tactic is to set $e_{n+1}=1$ and then compute $e_{i}=d_{i} \wedge\left(f_{i} \Rightarrow e_{i+1}\right)$ for $i \leq n$. This tactic is best explained by unfolding the definitions of $e_{n}$, then $e_{n-1}$, then $e_{n-2}$, and so on. This reverse ordering reflects the order in which the $e_{i}$ are computed; the $e_{i}$ are computed whilst walking backward across the clause. Any calling mode is safe for the empty goal and hence $e_{n+1}=1$. Note that $e_{n}=d_{n} \wedge\left(f_{n} \Rightarrow e_{n+1}\right)=d_{n} \wedge\left(\neg f_{n} \vee 1\right)=d_{n}$. Hence $e_{n}$ represents a safe calling mode for the goal $p_{n}\left(\vec{x}_{n}\right)$.

Observe that $e_{i}$ should not be larger than $d_{i}$, otherwise an error may occur while executing $p_{i}\left(\vec{x}_{i}\right)$. Observe too that if $p_{i}\left(\vec{x}_{i}\right), \ldots, p_{n}\left(\vec{x}_{n}\right)$ is called with a mode described by $d_{i}$, then $p_{i+1}\left(\vec{x}_{i+1}\right), \ldots, p_{n}\left(\vec{x}_{n}\right)$ is called with a mode described by $\left(d_{i} \wedge f_{i}\right)$ since $f_{i}$ describes the success patterns of $p_{i}\left(\vec{x}_{i}\right)$. The mode $\left(d_{i} \wedge f_{i}\right)$ may satisfy the $e_{i+1}$ demand. If it does not, then the minimal extra demand is added to $\left(d_{i} \wedge f_{i}\right)$ so as to satisfy $e_{i+1}$. This minimal extra demand is $\left(\left(d_{i} \wedge f_{i}\right) \Rightarrow e_{i+1}\right)-$ the weakest mode that, in conjunction with $\left(d_{i} \wedge f_{i}\right)$, ensures that $e_{i+1}$ holds. Put another way, $\left(\left(d_{i} \wedge f_{i}\right) \Rightarrow e_{i+1}\right)=\vee\left\{f \in \operatorname{Pos} \mid\left(d_{i} \wedge f_{i}\right) \wedge f \models e_{i+1}\right\}$.

Combining the requirements to satisfy $p_{i}\left(\vec{x}_{i}\right)$ and then $p_{i+1}\left(\vec{x}_{i+1}\right), \ldots, p_{n}\left(\vec{x}_{n}\right)$, gives $e_{i}=d_{i} \wedge\left(\left(d_{i} \wedge f_{i}\right) \Rightarrow e_{i+1}\right)$ which reduces to $e_{i}=d_{i} \wedge\left(f_{i} \Rightarrow e_{i+1}\right)$ and corresponds to the tactic used in the basic inductive step.

\subsubsection{Pseudo-complement}

This step of calculating the weakest mode that when conjoined with $d_{i} \wedge f_{i}$ implies $e_{i+1}$, is the very heart of the analysis. Setting $e_{i}=0$ would trivially achieve safety, but $e_{i}$ should be as weak as possible to maximise the class of safe queries inferred. For Pos, computing the weakest $e_{i}$ reduces to applying the $\Rightarrow$ operator, but more generally, this step amounts to applying the pseudo-complement operator. The pseudo-complement operator (if it exists for a given abstract domain) takes, as input, two abstractions and returns, as output, the weakest abstraction whose conjunction with the first input abstraction is at least as strong as the second input abstraction. If the domain did not possess a pseudo-complement, then there is not always a unique weakest abstraction (whose conjunction with one given abstraction is at least as strong as another given abstraction).

To see this, consider the domain Def (Armstrong et al., 1998) which does not possess a pseudo-complement. Def is the sub-class of Pos that is definite (Armstrong et al., 1998). This means that Def has the special property that each of its Boolean functions can be expressed as a (possibly empty) conjunction of propositional Horn clauses. As with Pos, Def is assumed to be augmented with the bottom element 0 . Def can thus represent the grounding dependencies $x \wedge y, x$, $x \Leftrightarrow y, y, x \Leftarrow y, x \Rightarrow y, 0$ and 1 but not $x \vee y$. Suppose that $d_{i} \wedge f_{i}=(x \Leftrightarrow y)$ and $e_{i+1}=(x \wedge y)$. Then conjoining $x$ with $d_{i} \wedge f_{i}$ would be at least as strong 
as $e_{i+1}$ and symmetrically conjoining $y$ with $d_{i} \wedge f_{i}$ would be at least as strong as $e_{i+1}$. However, Def does not contain a Boolean function strictly weaker than both $x$ and $y$, namely $x \vee y$, whose conjunction with $d_{i} \wedge f_{i}$ is at least as strong as $e_{i+1}$. Thus setting $e_{i}=x$ or $e_{i}=y$ would be safe but setting $e_{i}=(x \vee y)$ is prohibited because $x \vee y$ falls outside $D e f$. Moreover, setting $e_{i}=0$ would loose an unacceptable degree of precision. A choice would thus have to be made between setting $e_{i}=x$ and $e_{i}=y$ in some arbitrary fashion, so there would be no clear tactic for maximising precision.

Returning to the compound goal $p_{i}\left(\vec{x}_{i}\right), \ldots, p_{n}\left(\vec{x}_{n}\right)$, a call described by the mode $d_{i} \wedge\left(\left(d_{i} \wedge f_{i}\right) \Rightarrow e_{i+1}\right)$ is thus sufficient to ensure that neither $p_{i}\left(\vec{x}_{i}\right)$ nor the subgoal $p_{i+1}\left(\vec{x}_{i+1}\right), \ldots, p_{n}\left(\vec{x}_{n}\right)$ error. Since $d_{i} \wedge\left(\left(d_{i} \wedge f_{i}\right) \Rightarrow e_{i+1}\right)=d_{i} \wedge\left(f_{i} \Rightarrow e_{i+1}\right)$ $=e_{i}$ it follows that $p_{i}\left(\vec{x}_{i}\right), \ldots, p_{n}\left(\vec{x}_{n}\right)$ will not error if its call is described by $e_{i}$. In particular, it follows that $e_{1}$ describes a safe calling mode for the body atoms of the clause $p(\vec{x}) \leftarrow d \diamond f, p_{1}\left(\vec{x}_{1}\right), \ldots, p_{n}\left(\vec{x}_{n}\right)$.

The next step is to calculate $g=d \wedge\left(f \Rightarrow e_{1}\right)$. The abstraction $f$ describes the grounding behaviour of the Herbrand constraint added to the store prior to executing the body atoms. Thus $\left(f \Rightarrow e_{1}\right)$ describes the weakest mode that, in conjunction with $f$, ensures that $e_{1}$ holds, and hence the body atoms are called safely. Hence $d \wedge\left(f \Rightarrow e_{1}\right)$ represents the weakest demand that both satisfies the body atoms and the assertion $d$. One subtlety which relates to the abstraction process, is that $d$ is required to be a lower-approximation of the assertion whereas $f$ is required to be an upper-approximation of the constraint. Put another way, if the mode $d$ describes the binding on the store, then the (concrete) assertion is satisfied, whereas if the (concrete) constraint is added to the store, then the store is described by the mode $f$. Table 1 details how to abstract various builtins for groundness for a declarative subset of ISO Prolog.

\subsubsection{Strengthening lower approximations}

Variables not present in $p(\vec{x}), Y$ say, are then eliminated by $g^{\prime}=\forall_{Y}(g)$ (strengthening $g$ ) where $\forall_{\left\{y_{1} \ldots y_{n}\right\}}(g)=\forall_{y_{1}}\left(\ldots \forall_{y_{n}}(g)\right)$. A safe calling mode for this particular clause is then given by $g^{\prime}$. Eliminating variables from $g$ by strengthening $g$ is unusual and initially appears strange. Recall, however, that call patterns can be approximated from below without compromising correctness (but not from above). In particular the standard projection tactic of computing $\exists_{\left\{y_{1} \ldots y_{n}\right\}}(g)$ would result in an upper approximation of $g$ that possibly describes a larger set of concrete call patterns which would be incorrect. The direction of approximation thus dictates that eliminating the variables $Y$ from $g$ must strengthen $g$. Indeed, $g$ holds whenever $\forall_{y_{i}}(g)$ holds and therefore $g$ holds whenever $\forall_{\left\{y_{1} \ldots y_{n}\right\}}(g)$ holds as required.

$D_{k+1}$ will contain a call pattern $\left\langle p(\vec{x}), g^{\prime \prime}\right\rangle$ and, assuming $g^{\prime} \wedge g^{\prime \prime} \neq g^{\prime \prime}$, this is updated with $\left\langle p(\vec{x}), g^{\prime} \wedge g^{\prime \prime}\right\rangle$. Thus the call patterns become progressively stronger on each iteration. Correctness is preserved because call patterns can be safely approximated from below. The space of call patterns forms a complete lattice which ensures that a gfp exists. In fact, because call patterns are approximated from below, the gfp is the most precise solution, and therefore the desired solution. (This 
contrasts to the norm in logic program analysis where approximation is from above and the lfp is the most precise solution). Moreover, since the space of call patterns is finite, termination is assured. In fact, the scheme will converge onto the gfp since iteration commences with the top element $D_{0}=\{\langle p(\vec{x}), 1\rangle \mid p \in \Pi\}$.

\subsubsection{Greatest fixpoint calculation for Quicksort}

Under this procedure Quicksort generates the following $D_{k}$ sequence:

$$
\begin{array}{r}
D_{0}=\left\{\begin{array}{c}
\langle\mathrm{qs}(\vec{v}), 1\rangle \\
\langle\mathrm{pt}(\vec{w}), 1\rangle \\
\left\langle=<^{\prime}(\vec{u}), 1\right\rangle \\
\left\langle>^{\prime}(\vec{u}), 1\right\rangle
\end{array}\right\} D_{1}=\left\{\begin{array}{c}
\langle\mathrm{qs}(\vec{v}), 1\rangle \\
\langle\mathrm{pt}(\vec{w}), 1\rangle \\
\left\langle=<^{\prime}(\vec{u}), x_{1} \wedge x_{2}\right\rangle \\
\left\langle>^{\prime}(\vec{u}), x_{1} \wedge x_{2}\right\rangle
\end{array}\right\} \\
D_{2}=\left\{\begin{array}{c}
\langle\mathrm{qs}(\vec{v}), 1\rangle \\
\left\langle\operatorname{pt}(\vec{w}), x_{2} \wedge\left(x_{1} \vee\left(x_{3} \wedge x_{4}\right)\right)\right\rangle \\
\left\langle=<^{\prime}(\vec{u}), x_{1} \wedge x_{2}\right\rangle \\
\left\langle>^{\prime}(\vec{u}), x_{1} \wedge x_{2}\right\rangle
\end{array}\right\} D_{3}=\left\{\begin{array}{c}
\left\langle\mathrm{qs}(\vec{v}), x_{1}\right\rangle \\
\left\langle\mathrm{pt}(\vec{w}), x_{2} \wedge\left(x_{1} \vee\left(x_{3} \wedge x_{4}\right)\right)\right\rangle \\
\left\langle=<^{\prime}(\vec{u}), x_{1} \wedge x_{2}\right\rangle \\
\left\langle>^{\prime}(\vec{u}), x_{1} \wedge x_{2}\right\rangle
\end{array}\right\}
\end{array}
$$

These calculations are non-trivial so consider how $D_{2}$ is obtained from $D_{1}$ by applying the clause $\mathrm{pt}\left(t_{1}, m, t_{2}, h\right):-1 \diamond g_{4},=<^{\prime}(m, x), \operatorname{pt}(x s, m, l, h)$. The following $e_{i}$ and $g$ formulae are generated:

$$
\begin{array}{rlrl}
e_{3} & = & 1 & \\
e_{2} & =1 \wedge((x s \wedge l \wedge h) \Rightarrow 1)= & 1 \\
e_{1} & =(m \wedge x) \wedge((m \wedge x) \Rightarrow 1)= & m \wedge x \\
g & =1 \wedge\left(\left(\left(t_{1} \Leftrightarrow x \wedge x s\right) \wedge\left(t_{2} \Leftrightarrow x \wedge l\right)\right) \Rightarrow(m \wedge x)\right)
\end{array}
$$

To characterise those $\mathrm{pt}\left(t_{1}, m, t_{2}, h\right)$ calls which are safe, it is necessary to compute a function $g^{\prime}$ on the variables $t_{1}, m, t_{2}, h$ which, if satisfied by the mode of a call, ensures that $g$ is satisfied by the mode of the call. Put another way, it is necessary to eliminate the variables $x, x s$ and $l$ from $g$ (those variables which do not occur in the head $\left.\operatorname{pt}\left(t_{1}, m, t_{2}, h\right)\right)$ to strengthen $g$ obtain a function $g^{\prime}$ such that $g$ holds whenever $g^{\prime}$ holds. This is accomplished by calculating $g^{\prime}=\forall_{l} \forall_{x s} \forall_{x}(g)$. First consider the computation of $\forall_{x}(g)$ :

$$
\begin{aligned}
g[x \mapsto 0] & =\left(\left(\left(t_{1} \Leftrightarrow x \wedge x s\right) \wedge\left(t_{2} \Leftrightarrow x \wedge l\right)\right) \Rightarrow(m \wedge x)\right)[x \mapsto 0] \\
& =\left(\left(t_{1} \Leftrightarrow 0 \wedge x s\right) \wedge\left(t_{2} \Leftrightarrow 0 \wedge l\right)\right) \Rightarrow(m \wedge 0) \\
& =\left(\neg t_{1} \wedge \neg t_{2}\right) \Rightarrow 0 \\
& =t_{1} \vee t_{2} \\
g[x \mapsto 1] & =\left(\left(\left(t_{1} \Leftrightarrow x \wedge x s\right) \wedge\left(t_{2} \Leftrightarrow x \wedge l\right)\right) \Rightarrow(m \wedge x)\right)[x \mapsto 1] \\
& =\left(\left(t_{1} \Leftrightarrow x s\right) \wedge\left(t_{2} \Leftrightarrow l\right)\right) \Rightarrow m
\end{aligned}
$$

Since $g[x \mapsto 0] \wedge g[x \mapsto 1] \in$ Pos it follows that:

$$
\forall_{x}(g)=\left(\left(\left(t_{1} \Leftrightarrow x s\right) \wedge\left(t_{2} \Leftrightarrow l\right)\right) \Rightarrow m\right) \wedge\left(t_{1} \vee t_{2}\right)
$$


(otherwise $\forall_{x}(g)$ would be set to 0 ). Eliminating the other variables in a similar way we obtain:

$$
\begin{aligned}
\forall_{x s} \forall_{x}(g) & =\left(\left(t_{2} \Leftrightarrow l\right) \Rightarrow m\right) \wedge\left(t_{1} \vee t_{2}\right) \\
g^{\prime}=\forall_{l} \forall_{x s} \forall_{x}(g) & =m \wedge\left(t_{1} \vee t_{2}\right)
\end{aligned}
$$

Observe that if $\forall_{l} \forall_{x s} \forall_{x}(g)$ holds then $g$ holds. Thus if the mode of a call satisfies $g^{\prime}$ then the mode also satisfies $g$ as required. This clause thus yields the call pattern $\left\langle\operatorname{pt}(\vec{w}), x_{2} \wedge\left(x_{1} \vee x_{3}\right)\right\rangle$. Similarly the first and third clauses contribute the patterns $\langle\operatorname{pt}(\vec{w}), 1\rangle$ and $\left\langle\operatorname{pt}(\vec{w}) \leftarrow x_{2} \wedge\left(x_{1} \vee x_{4}\right)\right\rangle$. Observe also that

$$
1 \wedge\left(x_{2} \wedge\left(x_{1} \vee x_{3}\right)\right) \wedge\left(x_{2} \wedge\left(x_{1} \vee x_{4}\right)\right)=x_{2} \wedge\left(x_{1} \vee\left(x_{3} \wedge x_{4}\right)\right)
$$

which gives the final call pattern formula for $\operatorname{pt}(\vec{w})$ in $D_{2}$. The gfp is reached at $D_{3}$ since $D_{4}=D_{3}$. The gfp often expresses elaborate calling modes, for example, it states that $\operatorname{pt}(\vec{w})$ cannot generate an instantiation error (nor any predicate that it calls) if it is called with its second, third and fourth argument ground. This is a surprising result which suggests that the analysis can infer information that might be normally missed by a programmer.

\subsubsection{Restrictions posed by the framework}

The chief computational requirement of the analysis is that the input domain is equipped with a pseudo-complement operation. As already mentioned, it is always possible to systematically design a domain with this operator (Giacobazzi \& Scozzari, 1998) and any domain that is known to be condensing (see section 1.2) comes equipped with this operator. Currently, however, there are only a few domains with a pseudo-complement. Indeed, the domain described in (Codish \& Lagoon, 2000) appears to be unique in that it is the only type domain that is condensing. This is the main limitation of the backward analysis described in this paper.

$P o s$ is downward-closed in the sense that if a function $f$ describes a substitutions, then $f$ also describes all substitutions less general than the substitution. The type domain of (Codish \& Lagoon, 2000) is also downward-closed. It does not follow, however, that a domain equipped with a pseudo-complement operation is necessarily downward-closed. Heyting completion, the domain refinement technique used to construct pseudo-complement, can be moved to linear implication (Giacobazzi et al., 1998), though the machinery is more complicated. However, it is likely, that in the short term tractable condensing domains will continue to be downwardclosed. In fact, constructing tractable downward-closed condensing domains is a topic within itself.

\section{Preliminaries}

\subsection{Basic Concepts}

Sets and sequences Let $\mathbb{N}$ denote the set of non-negative integers. The powerset of $S$ is denoted $\wp(S)$. The empty sequence is denoted $\epsilon$ and $S^{\star}$ denotes the set of 
(possibly empty) sequences whose elements are drawn from $S$. Sequence concatenation is denoted $\cdot$ and the length of a sequence $s$ is $|s|$. Furthermore, let $s^{0}=\epsilon$ and $s^{n}=s \cdot s^{n-1}$ where $n \in \mathbb{N}$. If $n \in \mathbb{N}$ and $s \in \mathbb{N}^{\star}$ then $\max (n \cdot s)=\max (n, \max (s))$ where $\max (\epsilon)=0$.

Orderings A pre-order on a set $S$ is a binary relation $\sqsubseteq$ that is reflexive and transitive. A partial order on a set $S$ is a pre-order that is anti-symmetric. A poset $\langle S, \sqsubseteq\rangle$ is a partial order on a set $S$. If $\langle S, \sqsubseteq\rangle$ is a poset, then $C \subseteq S$ is a chain iff $a \sqsubseteq b$ or $b \sqsubseteq a$ for all $a, b \in C$. A meet semi-lattice $\langle L, \sqsubseteq, \sqcap\rangle$ is a poset $\langle L, \sqsubseteq\rangle$ such that the meet (greatest lower bound) $\sqcap\{x, y\}$ exists for all $x, y \in L$. A complete lattice is a poset $\langle L, \sqsubseteq\rangle$ such that the meet $\sqcap X$ and the join $\sqcup X$ (least upper bound) exist for all $X \subseteq L$. Top and bottom are respectively defined by $\top=\sqcap \emptyset$ and $\perp=\sqcup \emptyset$. A complete lattice is denoted $\langle L, \sqsubseteq, \sqcap, \sqcup, \top, \perp\rangle$. Let $\langle S, \sqsubseteq\rangle$ be a pre-order. If $X \subseteq S$ then $\downarrow(X)=\{y \in S \mid \exists x \in X . y \sqsubseteq x\}$. If $x \in S$ then $\downarrow(x)=\downarrow(\{x\})$. The set of orderideals of $S$, denoted $\wp^{\downarrow}(S)$, is defined by $\wp^{\downarrow}(S)=\{X \subseteq S \mid X=\downarrow(X)\}$. Observe that $\left\langle\wp^{\downarrow}(S), \subseteq, \cup, \cap, S, \emptyset\right\rangle$ is a complete lattice.

An algebraic structure is a pair $\langle S, \mathcal{Q}\rangle$ where $S$ is a non-empty set and $\mathcal{Q}$ is collection of $n$-ary operations $f: S^{n} \rightarrow S$ where $n \in \mathbb{N}$. Let $\langle S, \sqsubseteq\rangle$ and $\left\langle S^{\prime}, \sqsubseteq^{\prime}\right\rangle$ be posets and $\langle S, \mathcal{Q}\rangle$ and $\left\langle S^{\prime}, \mathcal{Q}^{\prime}\right\rangle$ algebraic structures such that $\mathcal{Q}=\left\{f_{i} \mid i \in I\right\}$ and $\mathcal{Q}^{\prime}=\left\{f_{i}^{\prime} \mid i \in I\right\}$ for an index set $I$. Then $\alpha: S \rightarrow S^{\prime}$ is a semi-morphism between $\langle S, \mathcal{Q}\rangle$ and $\left\langle S^{\prime}, \mathcal{Q}^{\prime}\right\rangle$ iff $\alpha\left(f_{i}\left(s_{1}, \ldots, s_{n}\right)\right) \sqsubseteq f_{i}^{\prime}\left(\alpha\left(s_{1}\right), \ldots, \alpha\left(s_{n}\right)\right)$ for all $\left\langle s_{1}, \ldots, s_{n}\right\rangle \in$ $S^{n}$ and $i \in I$.

Functions and fixpoints Let $f: A \rightarrow B$. Then $\operatorname{dom}(f)$ denotes the domain of $f$ and if $C \subseteq A$ then $f(C)=\{f(c) \mid c \in C\}$. Furthermore, $\operatorname{cod}(f)=f(\operatorname{dom}(f))$. Let $\langle L, \sqsubseteq, \sqcup, \sqcap\rangle$ and $\left\langle L^{\prime}, \sqsubseteq^{\prime}, \sqcup^{\prime}, \sqcap^{\prime}\right\rangle$ be complete lattices. The map $f: L \rightarrow L^{\prime}$ is additive iff $f(\sqcup X)=\sqcup^{\prime} f(X)$ for all $X \subseteq L ; f$ is continuous iff $f(\sqcup C)=\sqcup^{\prime} f(C)$ for all chains $C \subseteq L ; f$ is co-continuous iff $f(\sqcap C)=\sqcap^{\prime} f(C)$ for all chains $C \subseteq L$ and $f$ is monotonic iff $f(x) \sqsubseteq^{\prime} f(y)$ for all $x \sqsubseteq y$. Let $x \sqsubseteq y$. If $f$ is continuous then $f(y)=f(x \sqcup y)=\sqcup^{\prime}\{f(x), f(y)\}$ and thus $f(x) \sqsubseteq^{\prime} f(y)$. If $f$ is co-continuous then $f(x)=f(x \sqcap y)=\sqcap^{\prime}\{f(x), f(y)\}$ and thus $f(x) \sqsubseteq^{\prime} f(y)$. Both continuity and co-continuity thus imply monotonicity. If $f: L \rightarrow L$, then $f$ is idempotent iff $f(x)=f^{2}(x)$ for all $x \in L$ and $f$ is extensive iff $x \sqsubseteq f(x)$ for all $x \in L$. The Knaster-Tarski theorem states that any monotone operator $f: L \rightarrow L$ on a complete lattice $\langle L, \sqsubseteq, \sqcup, \sqcap, \top, \perp\rangle$ admit both greatest and least fixpoints that are characterised by $\operatorname{gfp}(f)=\sqcup\{x \in L \mid x \sqsubseteq f(x)\}$ and $\operatorname{lfp}(f)=\sqcap\{x \in L \mid f(x) \sqsubseteq x\}$. If $f$ is co-continuous then $\operatorname{gfp}(f)=\sqcap_{n \in \mathbb{N}} f^{n}(\top)$ and dually if $f$ is continuous then $\operatorname{lfp}(f)=\sqcup_{n \in \mathbb{N}} f^{n}(\perp) .\left\{f^{n}(\top) \mid n \in \mathbb{N}\right\}$ and $\left\{f^{n}(\perp) \mid n \in \mathbb{N}\right\}$ are, respectively, the lower and upper Kleene iteration sequences of $f$.

Galois insertions and closure operators If $\langle S, \sqsubseteq\rangle$ and $\left\langle S^{\prime}, \sqsubseteq^{\prime}\right\rangle$ are posets and $\alpha: S \rightarrow S^{\prime}$ and $\gamma: S^{\prime} \rightarrow S$ are monotonic maps such that $\forall x \in S . x \sqsubseteq \gamma(\alpha(x))$ and $\forall x^{\prime} \in S^{\prime} . \alpha\left(\gamma\left(x^{\prime}\right)\right) \sqsubseteq^{\prime} x^{\prime}$, then the quadruple $\left\langle S, \gamma, S^{\prime}, \alpha\right\rangle$ is a Galois connection between $S$ and $S^{\prime}$. In other words, $\alpha$ is the lower (or left) adjoint of $\gamma$ and $\gamma$ is the upper (or right) adjoint of $\alpha$. If, in addition, $\forall x^{\prime} \in S^{\prime} . x^{\prime} \sqsubseteq^{\prime} \alpha\left(\gamma\left(x^{\prime}\right)\right)$, then $\left\langle S, \gamma, S^{\prime}, \alpha\right\rangle$ 
is a Galois insertion between $S$ and $S^{\prime}$. The operator $\rho: L \rightarrow L$ on a complete lattice $\langle L, \sqsubseteq\rangle$ is a closure operator iff $\rho$ is monotonic, idempotent and extensive. The set of closure operators on $L$ is denoted $u c o(L)$. The image set $\rho(L)$ of a closure operator $\rho$ is a complete lattice with respect to $\sqsubseteq$. A Galois insertion $\left\langle L, \gamma, L^{\prime}, \alpha\right\rangle$ between the complete lattices $L$ and $L^{\prime}$ defines the closure operator $\rho=\gamma \circ \alpha$. Conversely, a closure operator $\rho: L \rightarrow L$ on the complete lattice $\langle L, \sqsubseteq, \sqcup\rangle$ defines the Galois insertion $\langle L, i d, \rho(L), \rho\rangle$ where $i d$ denotes identity. Galois insertions and closure operators are thus isomorphic, though closure operators are typically more succinct and hence used in this paper.

Substitutions Let Sub denote the set of (idempotent) substitutions and let Ren denote the set of (bijective) renaming substitutions.

\subsection{Cylindric constraint systems}

Let $V$ denote a (denumerable) universe of variables and let $\mathcal{C}$ denote a constraint system over $V$. An algebra $\left\langle\mathcal{C}, \unlhd, \otimes, 1,\left\{\exists_{x}\right\}_{x \in V},\left\{d_{x, y}\right\}_{x, y \in V}\right\rangle$ is a semi-cylindric constraint system iff $\langle\mathcal{C}, \unlhd, \otimes\rangle$ is a meet semi-lattice with a top $1 ; \exists_{x}$ is a family of (unary) cylindrification operations such that: $c \unlhd \exists_{x}(c), \exists_{x}(c) \unlhd \exists_{x}\left(c^{\prime}\right)$ if $c \unlhd c^{\prime}$, $\exists_{x}\left(c \otimes \exists_{x}\left(c^{\prime}\right)\right)=\exists_{x}(c) \otimes \exists_{x}\left(c^{\prime}\right)$; and $d_{x, y}$ is a family of (constant) diagonalisation operations such that: $d_{x, x}=1, d_{x, y}=\exists_{z}\left(d_{x, z} \otimes d_{z, y}\right)$ and $d_{x, y} \otimes \exists_{x}\left(c \otimes d_{x, y}\right) \unlhd c$ if $x \neq y$. Cylindrification captures the concept of projecting out a variable (and is useful in modeling variables that go out of scope) whereas diagonalisation captures the notion of an alias between two variables (and is useful in modeling parameter passing). (The reader is referred to (Giacobazzi et al., 1995) for further details on cylindric constraint systems and their application in abstract interpretation.)

\section{Example 3.1}

An equation $e$ is a pair $(s=t)$ where $s$ and $t$ are terms. A finite conjunction of equations is denoted $E$ and $E q n$ denotes the set of finite conjunctions of equations. Let $\operatorname{eqn}(\theta)=\{x=t \mid x \mapsto t \in \theta\}$ and unify $(E)=\{\theta \in S u b \mid \forall(s=$ $t) \in E \cdot \theta(s)=\theta(t)\}$. Eqn is pre-ordered by entailment $E_{1} \unlhd E_{2}$ iff unify $\left(E_{1}\right) \subseteq$ unify $\left(E_{2}\right)$ and quotiented by $E_{1} \approx E_{2}$ iff $E_{1} \unlhd E_{2}$ and $E_{2} \unlhd E_{1}$. This gives the meet semi-lattice $\langle E q n / \approx, \unlhd, \otimes\rangle$ with a top 1 where conjunction is defined $\left[E_{1}\right]_{\approx} \otimes\left[E_{2}\right]_{\approx}=\left[E_{1} \cup E_{2}\right]_{\approx}$ and $1=[\emptyset]_{\approx}$. Let $\operatorname{mgu}(E)=\{\theta \in$ unify $(E) \mid \forall \kappa \in$ unify $(E) . e q n(\kappa) \unlhd e q n(\theta)\}$. Finally, let $d_{x, y}=[\{x=y\}] \approx$ and define project out by $\exists_{x}\left([E]_{\approx}\right)=[e q n(\{y \mapsto t \in \theta \mid x \neq y\})] \approx$ if $\theta \in m g u(E)$. Otherwise, if $m g u(E)=\emptyset$, define $\exists_{x}\left([E]_{\approx}\right)=[\{a=b\}]_{\approx}$ where $a$ and $b$ are distinct constant symbols. Then $\left\langle E q n / \approx, \unlhd, \otimes, 1,\left\{\exists_{x}\right\}_{x \in V},\left\{d_{x, y}\right\}_{x, y \in V}\right\rangle$ is a semi-cylindric constraint system.

An algebra $\left\langle\mathcal{C}, \unlhd, \oplus, \otimes, 1,0,\left\{\exists_{x}\right\}_{x \in V},\left\{d_{x, y}\right\}_{x, y \in V}\right\rangle$ that extends a semi-cylindric constraint system to a complete lattice $\langle\mathcal{C}, \unlhd, \oplus, \otimes, 1,0\rangle$ is a cylindric constraint system. A semi-cylindric constraint system can be lifted to a cylindric constraint system via a power-domain construction. In particular $\left\langle\wp^{\downarrow}(\mathcal{C}), \subseteq, \cup, \cap, \mathcal{C}, \emptyset,\left\{\exists_{x}^{\prime}\right\}_{x \in V}\right.$, $\left.\left\{d_{x, y}^{\prime}\right\}_{x, y \in V}\right\rangle$ is a cylindric constraint system where $\exists_{x}^{\prime}(C)=\downarrow\left(\left\{\exists_{x}(c) \mid c \in C\right\}\right)$ and $d_{x, y}^{\prime}=\downarrow\left(d_{x, y}\right)$. 
Example 3.2

The semi-cylindric system of example 3.1 can be lifted to the cylindric system $\left\langle\wp^{\downarrow}(E q n), \subseteq, \cup, \cap, E q n, \emptyset, \exists^{\prime}, d^{\prime}\right\rangle$ where $\exists_{x}^{\prime}(C)=\downarrow\left(\left\{\exists_{x}(c) \mid c \in C\right\}\right)$ and $d_{x, y}^{\prime}=\downarrow\left(d_{x, y}\right)$.

In the sequel, unless otherwise stated, all constraint systems considered are over the same $V$ and thus a cylindric constraint system will be simply denoted $\langle\mathcal{C}, \unlhd, \oplus, \otimes, 1,0, \exists, d\rangle$. Let $\operatorname{var}(o)$ denote the set of the variables in the syntactic object $o$ and let $F V(c)$ denote the set of free variables in a constraint $c \in \mathcal{C}$, that is, $F V(c)=\left\{x \in \operatorname{var}(c) \mid \exists y \in V . c \neq \exists_{x}\left(c \otimes d_{x, y}\right)\right\}$. Abbreviate project out by $\exists_{\left\{x_{1}, \ldots, x_{n}\right\}}(c)=\exists_{x_{1}}\left(\ldots\left(\exists_{x_{n}}(c)\right)\right)$ and project onto by $\bar{\exists}_{X}(c)=\exists_{F V(c) \backslash X}(c)$. Let $d_{\vec{x}, \vec{y}}=\otimes_{i=1}^{n} d_{x_{i}, y_{i}}$ where $\vec{x}=\left\langle x_{1} \ldots x_{n}\right\rangle$ and $\vec{y}=\left\langle y_{1} \ldots y_{n}\right\rangle$. If $c \in \mathcal{C}$ then let $\partial_{\vec{x}}^{\vec{y}}(c)$ denote the constraint obtained by replacing $\vec{x}$ with $\vec{y}$, that is, $\partial_{\vec{x}}^{\vec{y}}(c)=$ $\exists_{z}\left(\exists_{\vec{x}}\left(c \otimes d_{\vec{x}, \vec{z})} \otimes d_{\vec{z}, \vec{y}}\right)\right.$ where $\operatorname{var}(\vec{z}) \cap(F V(c) \cup \operatorname{var}(\vec{x}) \cup \operatorname{var}(\vec{y}))=\emptyset$. Finally, if $C \subseteq \mathcal{C}$ then $\partial_{\vec{x}}^{\vec{y}}(C)=\left\{\partial_{\vec{x}}^{\vec{y}}(c) \mid c \in C\right\}$.

\section{Example 3.3}

Let $X$ be a finite subset of $V$. The groundness domain $\left\langle E P \operatorname{Pos}_{X}, \models, \curlyvee, \wedge, 1,0\right\rangle$ (Heaton et al., 2000) is a finite lattice where $\operatorname{EPos}_{X}=\{0\} \cup\left\{\wedge F \mid F \subseteq X \cup E_{X}\right\}$, $E_{X}=\{x \Leftrightarrow y \mid x, y \in X\}$ and $f_{1} \curlyvee f_{2}=\wedge\left\{f \in E_{\operatorname{Pos}_{X}} \mid f_{1} \models f \wedge f_{2} \models f\right\} . E^{2} \operatorname{Pos}_{X}$ is a cylindric constraint system with $d_{x, y}=(x \Leftrightarrow y)$ and $\exists_{x}(f)=f^{\prime} \wedge f^{\prime \prime}$ where $f^{\prime}=\wedge\{y \in Y \mid f \models y\}, f^{\prime \prime}=\wedge\left\{e \in E_{Y} \mid f \models e\right\}$ and $Y=X \backslash\{x\}$.

Example 3.4

Let $\mathrm{Bool}_{X}$ denote the Boolean functions over $X$. The dependency domain $\operatorname{Pos}_{X}$ (Armstrong et al., 1998) is defined by $\operatorname{Pos}_{X}=\{0\} \cup\left\{f \in B_{0 o l_{X}} \mid \wedge X \models f\right\}$. Henceforth $Y$ abbreviates $\wedge Y$. The lattice $\left\langle\operatorname{Pos}_{X}, \models, \vee, \wedge, 1,0\right\rangle$ is finite and is a cylindric constraint system with $d_{x, y}=(x \Leftrightarrow y)$ and Schröder elimination defining $\exists_{x}(f)=f[x \mapsto 1] \vee f[x \mapsto 0]$.

\subsection{Complete Heyting algebras}

Let $\langle L, \sqsubseteq, \sqcap\rangle$ be a lattice with $x, y \in L$. The pseudo-complement of $x$ relatively to $y$, if it exists, is a unique element $z \in L$ such that $x \sqcap w \sqsubseteq y$ iff $w \sqsubseteq z . L$ is relatively pseudo-completed iff the pseudo-complement of $x$ relative to $y$, denoted $x \rightarrow y$, exists for all $x, y \in L$. If $L$ is also complete then it is a complete Heyting algebra (cHa). If $x, y \in L$ then $x \sqcap(x \rightarrow y)=x \sqcap y$. Furthermore, if $\langle L, \sqsubseteq, \sqcup, \sqcap\rangle$ is a cHa then $x \rightarrow y=\sqcup\{w \in L \mid x \sqcap w \sqsubseteq y\}$. The intuition behind the pseudo-complement of $x$ relative to $y$ is that it is the weakest element whose combination (meet) with $x$ implies $y$. Interestingly pseudo-complement can be interpreted as the adjoint of conjunction. (The reader is referred to (van Dalen, 1997) for further details on complete Heyting algebras.) The following result (Birkhoff, 1967)[Chapter IX, Theorem 15] explains how a cHa depends on the additivity of meet.

Theorem 3.1

A complete lattice $L$ is relatively pseudo-complemented iff $x \sqcap(\sqcup Y)=\sqcup\{x \sqcap y \mid$ $y \in Y\}$ for all $x \in L$ and $Y \subseteq L$.

Example 3.5 
Let $\{x, y\} \subseteq X$ and $f=(x \Leftrightarrow y)$. Then returning to EPos ${ }_{X}$ of example 3.3, $f \wedge(\curlyvee\{x, y\})=f \wedge(1)=f \neq(x \wedge y)=\curlyvee\{x \wedge y, x \wedge y\}=\curlyvee\{f \wedge x, f \wedge y\}$. Hence, by theorem 3.1, EPos ${ }_{X}$ is not a cHa. Now consider $\operatorname{Pos}_{X}$ of example 3.4, and specifically let $f \in \operatorname{Pos}_{X}$ and $G \subseteq \operatorname{Pos}_{X}$. Since $\wedge$ distributes over $\vee$, it follows that $f \sqcap(\sqcup G)=\sqcup\{f \sqcap g \mid g \in G\}$, thus by theorem 3.1, Pos $_{X}$ is a cHa. Similarly, $\cap$ distributes over $\cup$, and thus it follows by theorem 3.1 that $\wp^{\downarrow}(\mathcal{C})$ is also a cHa.

\subsection{Constraint logic programs}

Let $\Pi$ denote a (finite) set of predicate symbols, let Atom denote the set of (flat) atoms over $\Pi$ with distinct arguments drawn from $V$, and let $\langle\mathcal{C}, \unlhd, \oplus, \otimes, 1,0, \exists, d\rangle$ be a semi-cylindric constraint system. The set of constrained atoms is defined by Base $^{\mathcal{C}}=\{p(\vec{x}):-c \mid p(\vec{x}) \in$ Atom $\wedge c \in \mathcal{C}\}$. Let $F V(p(\vec{x}):-c)=\operatorname{var}(\vec{x}) \cup F V(c)$. Entailment $\unlhd$ lifts to Base $e^{\mathcal{C}}$ by $w_{1} \unlhd w_{2}$ iff $\bar{\exists}_{\vec{x}}\left(d_{\vec{x}, \vec{x}_{1}} \otimes c_{1}\right) \unlhd \bar{\exists}_{\vec{x}}\left(d_{\vec{x}, \vec{x}_{2}} \otimes c_{2}\right)$ where $w_{i}=p\left(\vec{x}_{i}\right):-c_{i}$ and $\operatorname{var}(\vec{x}) \cap\left(F V\left(w_{1}\right) \cup F V\left(w_{2}\right)\right)=\emptyset$. This pre-order defines the equivalence relation $w_{1} \approx w_{2}$ iff $w_{1} \unlhd w_{2}$ and $w_{2} \unlhd w_{1}$ to give a set of interpretations defined by $I n t^{\mathcal{C}}=\wp\left(\right.$ Base $\left.^{\mathcal{C}} / \approx\right)$. Int $t^{\mathcal{C}}$ is ordered by $I_{1} \sqsubseteq I_{2}$ iff for all $\left[w_{1}\right]_{\approx} \in I_{1}$ there exists $\left[w_{2}\right]_{\approx} \in I_{2}$ such that $w_{1} \unlhd w_{2}$. Let $\equiv$ denote the induced equivalence relation $I_{1} \equiv I_{2}$ iff $I_{1} \sqsubseteq I_{2}$ and $I_{2} \sqsubseteq I_{1} \cdot\left\langle I_{n} t^{\mathcal{C}} / \equiv, \sqsubseteq, \sqcup, \sqcap, \top, \perp\right\rangle$ is a complete lattice where $\left[I_{1}\right]_{\equiv} \sqcup\left[I_{2}\right]_{\equiv}=\left[I_{1} \cup I_{2}\right]_{\equiv},\left[I_{1}\right]_{\equiv} \sqcap\left[I_{2}\right]_{\equiv}=\left[\cup\left\{I \mid I \sqsubseteq I_{1} \wedge I \sqsubseteq I_{2}\right\}\right]_{\approx}$, $\top=\left[\left\{[p(\vec{x}):-1]_{\approx} \mid p(\vec{x}) \in \text { Atom }\right\}\right]_{\equiv}$ and $\perp=[\emptyset]_{\equiv}$.

A constraint logic program $P$ over $\mathcal{C}$ is a finite set of clauses $w$ of the form $w=h:-c, g$ where $h \in$ Atom, $c \in \mathcal{C}, g \in$ Goal and Goal $=$ Atom $^{\star}$. The fixpoint semantics of $P$ is defined in terms of an immediate consequences operator $\mathcal{F}_{P}^{\mathcal{C}}$.

\section{Definition 3.1}

Given a constraint logic program $P$ over a semi-cylindric constraint system $\mathcal{C}$, the operator $\mathcal{F}_{P}^{\mathcal{C}}: \operatorname{Int} t^{\mathcal{C}} \rightarrow \operatorname{Int}^{\mathcal{C}}$ is defined by:

$$
\mathcal{F}_{P}^{\mathcal{C}}(I)=\left\{\begin{array}{l|l}
{\left[p(\vec{x}):-c^{\prime}\right] \approx} & \begin{array}{cc}
\exists & p(\vec{x}):-c, p_{1}\left(\vec{x}_{1}\right), \ldots, p_{n}\left(\vec{x}_{n}\right) \in P \\
\exists & \left\{\left[p_{i}\left(\vec{x}_{i}\right):-c_{i}\right]_{\approx}\right\}_{i=1}^{n} \subseteq I \\
c^{\prime}=c \otimes \otimes_{i=1}^{n} \vec{\exists}_{\overrightarrow{x_{i}}}\left(c_{i}\right)
\end{array}
\end{array}\right\}
$$

The operator $\mathcal{F}_{P}^{\mathcal{C}}$ lifts to $\operatorname{Int} t^{\mathcal{C}} / \equiv$ by $\mathcal{F}_{P}^{\mathcal{C}}\left([I]_{\equiv}\right)=\left[\mathcal{F}_{P}^{\mathcal{C}}(I)\right]_{\equiv}$. The lifting is monotonic and hence the fixpoint semantics for a program $P$ over $\mathcal{C}$ exists and is denoted $\mathcal{F}^{\mathcal{C}}(P)=\operatorname{lfp}\left(\mathcal{F}_{P}^{\mathcal{C}}\right)$. (The reader is referred to (Bossi et al., 1994; Jaffar \& Maher, 1994) for further details on semantics and constraint logic programming.)

The operational semantics of $P$ is defined in terms of a transition system $\rightarrow_{P}$ between states of the form State $=$ Goal $\times \mathcal{C}$. To define the transition system, let $F V(\langle g ; c\rangle)=\operatorname{var}(g) \cup F V(c)$ and $F V(h:-c, g)=\operatorname{var}(h) \cup F V(c) \cup \operatorname{var}(g)$. To rename clauses with $\varphi \in$ Ren it is necessary to rename constraints with $\varphi$. Thus define $\varphi(h:-c, g)=\varphi(h):-\partial_{\vec{x}}^{\varphi(\vec{x})}(c), \varphi(g)$. To rename apart from a syntactic object $o$, let $w \ll_{o} P$ indicate that there exists $w^{\prime} \in P$ and $\varphi \in$ Ren such that $\operatorname{var}(\operatorname{cod}(\varphi)) \cap$ $F V\left(w^{\prime}\right)=\emptyset, \varphi\left(w^{\prime}\right)=w$ and $F V(o) \cap F V(w)=\emptyset$.

Definition 3.2 
Given a constraint logic program $P$ over a semi-cylindric constraint system $\mathcal{C}$, $\rightarrow_{P} \subseteq$ State $^{2}$ is the least relation such that:

$$
s=\langle p(\vec{x}), g ; c\rangle \rightarrow_{P}\left\langle g^{\prime}, g ; c \otimes d_{\vec{x}, \vec{x}^{\prime}} \otimes c^{\prime}\right\rangle
$$

where $p\left(\vec{x}^{\prime}\right):-c^{\prime}, g^{\prime} \ll_{s} P$.

The operational semantics is specified by the transitive closure of the transition relation on (atomic) goals, that is, $\mathcal{O}^{\mathcal{C}}(P)=\left[\left\{[p(\vec{x}):-c] \approx \mid\langle p(\vec{x}) ; 1\rangle \rightarrow_{P}^{\star}\langle\epsilon ; c\rangle\right\}\right]_{\equiv}$. The relationship between the operational and fixpoint semantics is stated below.

Theorem 3.2

$\mathcal{O}^{\mathcal{C}}(P)=\mathcal{F}^{\mathcal{C}}(P)$.

\subsection{Abstract semantics for constraint logic programs}

To apply abstraction techniques and finitely characterise $\mathcal{F}^{\mathcal{C}}(P)$, and thereby $\mathcal{O}^{\mathcal{C}}(P)$, the semi-cylindric domain $\mathcal{C}$ is replaced by the cHa $\wp^{\downarrow}(\mathcal{C})$ which is particularly amenable to approximation and backward reasoning.

If $P$ is a constraint logic program over $\mathcal{C}$, then $\downarrow(P)=\{h:-\downarrow(c), g \mid h:-c, g \in P\}$. Furthermore, if $I \in$ Int $^{\mathcal{C}}$, then let $\downarrow\left([I]_{\equiv}\right)=\left[\left\{[p(\vec{x}):-\downarrow(c)]_{\approx} \mid[p(\vec{x}):-c]_{\approx} \in I\right\}\right]_{\equiv}$. Note the overloading on $\approx$ and hence $\equiv$. The $\approx$ of $[p(\vec{x}):-c] \approx$ is induced by $\langle\mathcal{C}, \unlhd\rangle$ whereas the $\approx$ of $[p(\vec{x}):-\downarrow(c)] \approx$ is induced by $\left\langle\wp^{\downarrow}(\mathcal{C}), \subseteq\right\rangle$. The following proposition details the relationship between $\mathcal{F}^{\mathcal{C}}$ and $\mathcal{F}^{\wp^{\perp}}(\mathcal{C})$.

Proposition 3.1

$\downarrow\left(\mathcal{F}^{\mathcal{C}}(P)\right) \sqsubseteq \mathcal{F}^{\downarrow}(\mathcal{C})(\downarrow(P))$.

Let $\langle\mathcal{C}, \unlhd, \oplus, \otimes, 1,0, \exists, d\rangle$ denote a cylindric constraint system. If $\rho \in u c o(\mathcal{C})$ then $\langle\rho(\mathcal{C}), \unlhd, \otimes\rangle$ is a complete lattice. If $\rho$ is additive, then $\langle\rho(\mathcal{C}), \unlhd, \oplus, \otimes\rangle$ is a sub-lattice of $\langle\mathcal{C}, \unlhd, \oplus, \otimes\rangle$. More generally, the join is denoted $\oplus^{\prime}$. Observe that $\rho(\mathcal{C})$ has 1 and $\rho(0)$ for top and bottom and $c_{1} \oplus c_{2} \unlhd \rho\left(c_{1} \oplus c_{2}\right)=c_{1} \oplus^{\prime} c_{2}$ for all $c_{1}, c_{2} \in \rho(\mathcal{C})$. A cylindric constraint system is obtained by augmenting $\rho(\mathcal{C})$ with cylindrification $\exists_{x}^{\prime}$ and diagonalisation $d_{x, y}^{\prime}$ operators. To abstract $\langle\mathcal{C}, \unlhd, \oplus, \otimes, 1,0, \exists, d\rangle$ safely with $\left\langle\rho(\mathcal{C}), \unlhd, \oplus^{\prime}, \otimes, 1, \rho(0), \exists^{\prime}, d^{\prime}\right\rangle, \rho$ is required to be a semi-morphism (Giacobazzi et al., 1995) which additionally requires that $\rho\left(\exists_{x}(c)\right) \unlhd \exists_{x}^{\prime}(\rho(c))$ for all $c \in \mathcal{C}$ and $\rho\left(d_{x, y}\right) \unlhd d_{x, y}^{\prime}$ for all $x, y \in V$. In fact, these requirements turn out to be relatively weak conditions: most abstract domains come equipped with (abstract) operators to model projection and parameter passing.

\section{Example 3.6}

Consider the cylindric system $\left\langle\wp^{\downarrow}(E q n), \subseteq, \cup, \cap, E q n, \emptyset, \exists, d\right\rangle$ derived from the semicylindric system introduced in example 3.1. Let Bool $=\mathrm{Bool}_{V}$ and $\mathrm{Pos}=\mathrm{Pos}_{V}$. Define $\alpha_{\text {Pos }}: \wp^{\downarrow}(E q n) \rightarrow$ Pos by $\alpha_{\text {Pos }}(C)=\vee\{\alpha(\theta) \mid \theta \in m g u(E) \wedge E \in C\}$ and $\alpha(\theta)=\wedge\{x \Leftrightarrow \operatorname{var}(t) \mid x \mapsto t \in \theta\}$. Also define $\gamma_{P o s}:$ Pos $\rightarrow \wp^{\downarrow}(E q n)$ by $\gamma_{\text {Pos }}(f)=\cup\left\{C \in \wp^{\downarrow}(E q n) \mid \alpha_{\text {Pos }}(C) \models f\right\}$ and observe $\rho_{\text {Pos }} \in u c o\left(\wp^{\downarrow}(E q n)\right)$ where $\rho_{P o s}=\gamma_{P o s} \circ \alpha_{P o s}$. To construct a semi-morphism, put $d_{x, y}^{\prime}=\gamma_{P o s}(x \Leftrightarrow y)$ and $\exists_{x}^{\prime}(C)=\gamma_{\text {Pos }}(f[x \mapsto 1] \vee f[x \mapsto 0])$ where $f=\alpha_{\text {Pos }}(C)$. Then $\rho_{\text {Pos }}\left(d_{x, y}\right) \subseteq d_{x, y}^{\prime}$ and 
$\rho_{\text {Pos }}\left(\exists_{x}(C)\right) \subseteq \exists_{x}^{\prime}\left(\rho_{\text {Pos }}(C)\right)$ for all $C \in \wp^{\downarrow}(E q n)$. Note that $C_{1} \cap C_{2}=\gamma_{P o s}\left(f_{1} \wedge f_{2}\right)$ and $C_{1} \oplus^{\prime} C_{2}=\gamma_{P o s}\left(f_{1} \vee f_{2}\right)$ where $C_{i}=\gamma_{P o s}\left(f_{i}\right)$. Surprisingly $C_{1} \oplus^{\prime} C_{2} \neq C_{1} \cup C_{2}$ (Filé \& Ranzato, 1994), as witnessed by $C_{1}=\gamma_{P o s}(x)$ and $C_{2}=\gamma_{P o s}(x \Leftrightarrow y)$ since $\{y=f(x, z)\} \notin C_{1} \cup C_{2}$ whereas $\alpha_{P o s}(\{y=f(x, z)\})=y \Leftrightarrow(x \wedge z) \vDash x \vee(x \Leftrightarrow y)$ so that $\{y=f(x, z)\} \in C_{1} \oplus^{\prime} C_{2}=\gamma_{P o s}(x \vee(x \Leftrightarrow y))$. Nevertheless, $\rho_{P o s}$ is a semi-morphism between $\left\langle\wp^{\downarrow}(E q n), \subseteq, \cup, \cap, E q n, \emptyset, \exists, d\right\rangle$ and $\left\langle\rho_{P o s}\left(\wp^{\downarrow}(E q n)\right), \subseteq\right.$ $\left., \oplus^{\prime}, \cap, E q n, \rho_{P o s}(\emptyset), \exists^{\prime}, d^{\prime}\right\rangle$.

The operator $\rho$ lifts to the complete lattice $\operatorname{Int} t^{\mathcal{C}} / \equiv$ by $\rho\left([I]_{\equiv}\right)=[\rho(I)]_{\equiv}$ where $\rho(I)=\left\{[p(\vec{x}):-\rho(c)]_{\approx} \mid[p(\vec{x}):-c] \approx \in I\right\}$. Thus $\rho \in u c o\left(\operatorname{Int} t^{\mathcal{C}} / \equiv\right)$. It is also useful to lift $\rho$ to programs by $\rho(P)=\{h:-\rho(c), g \mid h:-c, g \in P\}$. The following result relates the fixpoint semantics of $P$ to that of its abstraction $\rho(P)$.

Theorem 3.3

Let $\mathcal{C}$ be a cylindric constraint system. If $\rho \in u c o(\mathcal{C})$ is a semi-morphism, then $\rho\left(\mathcal{F}^{\mathcal{C}}(P)\right) \sqsubseteq \mathcal{F}^{\operatorname{cod}(\rho)}(\rho(P))$.

Corollary 3.1

Let $\mathcal{C}$ be a semi-cylindric constraint system. If $\rho \in u c o\left(\wp^{\downarrow}(\mathcal{C})\right)$ is a semi-morphism, then $\rho\left(\downarrow\left(\mathcal{F}^{\mathcal{C}}(P)\right)\right) \sqsubseteq \mathcal{F}^{\operatorname{cod}(\rho)}(\rho(\downarrow(P)))$.

\section{Constraint logic programs with assertions}

We consider programs annotated with assertions (Drabent \& Małuszyński, 1988). When considering the operational semantics of a constraint logic program, it is natural to associate assertions with syntactic elements of the program such as predicates or the program points between body atoms. Without loss of generality, we decorate the neck of each clause with a set of constraints $C$ that is interpreted as an assertion. When $C$ is encountered, the store $c$ is examined to determine whether $c \in C$ (modulo renaming). If $c \in C$ execution proceeds normally, otherwise an error state, denoted $\diamond$, is entered and execution halts.

To formalise this idea, let $\mathcal{C}$ be a semi-cylindric constraint system and $\rho \in u \operatorname{co}\left(\wp^{\downarrow}(\mathcal{C})\right)$. The assertion language (in whatever syntactic form it takes) is described by $\rho$. A clause of a constraint logic program over $\mathcal{C}$ with assertions over $\operatorname{cod}(\rho)$ then takes the form $h:-C \diamond c, g$ where $h \in$ Atom, $C \in \operatorname{cod}(\rho), c \in \mathcal{C}, g \in$ Goal and $\diamond$ separates the assertion from the body of the clause. Notice that $C$ is an orderideal and thus downward closed. ( $C$ can thus represent disjunctions of constraints, but the semantics presented in this section should not be confused with a collecting semantics.) Note also that program transformation (Puebla et al., 2000a) can be used to express program point assertions in terms of our assertion language. To specify the behaviour of programs with assertions, let State $\diamond=$ State $\cup\{\diamond\}$, and let $\operatorname{CLP}(P)=\{h:-c, g \mid h:-C \diamond c, g \in P\}$. The following definition details how the operational semantics for the assertion language is realised in terms of projection, renaming and a test for inclusion.

Definition 4.1 
Given a constraint logic program $P$ over a semi-cylindric constraint system $\mathcal{C}$ with assertions over $\rho\left(\wp^{\downarrow}(\mathcal{C})\right), \Rightarrow_{P} \subseteq$ State $\times$ State $\diamond$ is the least relation such that:

$$
s=\langle p(\vec{x}), g ; c\rangle \Rightarrow_{P} \begin{cases}\diamond & \text { if } p\left(\vec{x}^{\prime}\right):-C^{\prime} \diamond c^{\prime}, g^{\prime} \in P \\ & \wedge \vec{x}_{\vec{x}}\left(\bar{\exists}_{\vec{x}}(c)\right) \notin C^{\prime} \\ \left\langle g^{\prime}, g ; c \otimes d_{\vec{x}, \vec{x}^{\prime}} \otimes c^{\prime}\right\rangle & \text { else if } p\left(\vec{x}^{\prime}\right):-c^{\prime}, g^{\prime} \ll_{s} \operatorname{CLP}(P)\end{cases}
$$

Recall that $p\left(\vec{x}^{\prime}\right):-c^{\prime}, g^{\prime} \ll_{s} \operatorname{CLP}(P)$ ensures that the clause $p\left(\vec{x}^{\prime}\right):-c^{\prime}, g^{\prime}$ does not share any variables with $s$. The operational semantics of $P$ is then defined in terms of $\Rightarrow_{P}^{\star}$ as $\mathcal{A}^{\rho, \mathcal{C}}(P)=\left[\left\{[p(\vec{x}):-c]_{\approx} \mid\langle p(\vec{x}) ; 1\rangle \Rightarrow_{P}^{\star}\langle\epsilon ; c\rangle\right\}\right]_{\equiv \text {. The relationship between }}$ two operational semantics is stated in the following (trivial) result.

\section{Proposition 4.1}

$\mathcal{A}^{\rho, \mathcal{C}}(P) \sqsubseteq \mathcal{O}^{\mathcal{C}}(\mathrm{CLP}(P))$

Assertions are often used as interface between behaviour that is amenable to formalisation, for example as an operational semantics, and behaviour that is less tractable, for example, the semantics of a builtin (Puebla et al., 2000b). More to the point, it is not always possible to infer the behaviour of a builtin from its definition, partly because builtins are often complicated and partly because builtins are often expressed in a language such as C. Our work requires assertions for each builtin in order to specify: its calling convention (for example, which arguments are required to be ground) and its success behaviour (for example, which arguments are grounded).

\section{Backward fixpoint semantics for constraint logic programs with assertions}

Let $P$ be a constraint logic program over the semi-cylindric constraint system $\mathcal{C}$ with assertions over $\rho\left(\wp^{\downarrow}(\mathcal{C})\right)$. One natural and interesting question is whether the error state $\diamond$ is reachable (or conversely not reachable) in $P$ from an initial state $\langle p(\vec{x}) ; c\rangle$. For a given constraint logic program $P$ with assertions, the backward fixpoint semantics presented in this section infers a (possibly empty) set of $c \in \mathcal{C}$ for which $\langle p(\vec{x}) ; c\rangle \nRightarrow{ }_{P}^{\star} \oslash$. The semantics formalises the informal backward analysis sketched in section 2 .

For generality, the semantics is parameterised by $\mathcal{C}$ and $\rho$. The correctness argument requires $\rho$ to be a semi-morphism between $\left\langle\wp^{\downarrow}(\mathcal{C}), \subseteq, \cup, \cap, \mathcal{C}, \emptyset, \exists, d\right\rangle$ and $\left\langle\rho\left(\wp^{\downarrow}(\mathcal{C})\right), \subseteq, \oplus^{\prime}, \cap, \mathcal{C}, \rho(\emptyset), \exists^{\prime}, d^{\prime}\right\rangle$. Additionally, $\rho\left(\wp^{\downarrow}(\mathcal{C})\right)$ must be a cHa, that is, it must possess a pseudo-complement $\rightarrow^{\prime}$. To explain, how pseudo-complement aids backward analysis consider the problem of inferring $c \in \mathcal{C}$ for which $\langle g ; c\rangle \not{ }_{P}^{\star} \diamond$ where $g=p_{1}\left(\vec{x}_{1}\right), \ldots, p_{n}\left(\vec{x}_{n}\right)$. Suppose $f_{i} \in \rho\left(\wp^{\downarrow}(\mathcal{C})\right)$ describes the success pattern for $p_{i}\left(\vec{x}_{i}\right)$, that is, if $\left\langle p_{i}\left(\vec{x}_{i}\right) ; 1\right\rangle \rightarrow_{P}^{\star}\langle\epsilon ; c\rangle$ then $c \in f_{i}$. Moreover, suppose $d_{i} \in \rho\left(\wp^{\downarrow}(\mathcal{C})\right)$ approximates the initial call pattern for $p_{i}\left(\vec{x}_{i}\right)$, that is, if $c \in d_{i}$ then $\left\langle p_{i}\left(\vec{x}_{i}\right) ; c\right\rangle \nRightarrow_{P}^{\star} \diamond$. Observe that $\left\langle p_{n-1}\left(\vec{x}_{n-1}\right), p_{n}\left(\vec{x}_{n}\right) ; c\right\rangle \nRightarrow_{P}^{\star} \diamond$ if $c \in d_{n-1} \cap e$ and $e \cap\left(d_{n-1} \cap f_{n-1}\right) \subseteq d_{n}$. This follows since $\left\langle p_{n-1}\left(\vec{x}_{n-1}\right) ; c\right\rangle \not \nexists_{P}^{\star} \diamond$ because $c \in d_{n-1} \cap e \subseteq d_{n-1}$. Moreover, if $\left\langle p_{n-1}\left(\vec{x}_{n-1}\right), p_{n}\left(\vec{x}_{n}\right) ; c\right\rangle \Rightarrow_{P}^{\star}\left\langle p_{n}\left(\vec{x}_{n}\right) ; c^{\prime}\right\rangle$ then $c^{\prime} \in\left(d_{n-1} \cap e\right) \cap f_{n-1} \subseteq d_{n}$ and thus $\left\langle p_{n}\left(\vec{x}_{n}\right) ; c^{\prime}\right\rangle \nRightarrow_{P}^{\star} \diamond$. Putting $e=\rho(\emptyset)$ ensures 
$e \cap\left(d_{n-1} \cap f_{n-1}\right) \subseteq d_{n}$ and thereby achieves correctness. However, for precision, $d_{n-1} \cap e$ should be maximised. Since $\rho\left(\wp^{\downarrow}(\mathcal{C})\right)$ is a cHa, this reduces to assigning $e=\oplus^{\prime}\left\{e^{\prime} \in \rho\left(\wp^{\downarrow}(\mathcal{C})\right) \mid e^{\prime} \cap\left(d_{n-1} \cap f_{n-1}\right) \subseteq d_{n}\right\}=\left(d_{n-1} \cap f_{n-1}\right) \rightarrow^{\prime} d_{n}$. In general, without pseudo-complement, there is no unique best $e$ that maximises precision (see example 5.1). The construction is generalised for $g=p_{1}\left(\vec{x}_{1}\right), \ldots, p_{n}\left(\vec{x}_{n}\right)$, by putting $e_{n}=\mathcal{C}$ and $e_{i}=d_{i} \cap\left(\left(d_{i} \cap f_{i}\right) \rightarrow^{\prime} e_{i+1}\right)=d_{i} \cap\left(f_{i} \rightarrow^{\prime} e_{i+1}\right)$ for $1 \leq i<n$. Then $\langle g ; c\rangle \nRightarrow_{P}^{\star} \diamond$ if $c \in e_{1}$ as required. This iterated application of $\rightarrow^{\prime}$ to propagate requirements right-to-left is the very essence of the backward analysis.

\section{Example 5.1}

Returning to examples 3.2-3.5, let $\alpha_{E P o s}(C)=\curlyvee\{\alpha(\theta) \mid \theta \in m g u(E) \wedge E \in C\}$, $\gamma_{E P o s}(f)=\cup\left\{C \in \wp^{\downarrow}(E q n) \mid \alpha_{E P o s}(C) \vDash f\right\}$ and $\rho_{E P o s}=\gamma_{E P o s} \circ \alpha_{E P o s}$. Note that $C_{1} \cap C_{2}=\gamma_{E P o s}\left(f_{1} \wedge f_{2}\right)$ and $C_{1} \oplus^{\prime} C_{2}=\gamma_{E P o s}\left(f_{1} \curlyvee f_{2}\right)$ where $C_{i}=\gamma_{E P o s}\left(f_{i}\right)$. By defining $\exists^{\prime}$ and $d^{\prime}$ in an analogous way to example 3.5 , a semi-morphism $\rho_{E P o s}$ is constructed between $\left\langle\wp^{\downarrow}(E q n), \subseteq, \cup, \cap, E q n, \emptyset, \exists, d\right\rangle$ and $\left\langle\rho_{E P o s}\left(\wp^{\downarrow}(E q n)\right), \subseteq, \oplus^{\prime}, \cap, E q n, \rho_{E P o s}(\emptyset), \exists^{\prime}, d^{\prime}\right\rangle$. Recall that $\rho_{E P o s}\left(\wp^{\downarrow}(E q n)\right)$ is not a cHa. Now consider the problem of inferring an initial $c$ for $\left\langle p_{n-1}\left(\vec{x}_{n-1}\right), p_{n}\left(\vec{x}_{n}\right) ; c\right\rangle$ within $\rho_{E P o s}\left(\wp^{\downarrow}(E q n)\right)$. In particular let $d_{n-1}=\gamma_{E P o s}(1), f_{n-1}=\gamma_{E P o s}(x \Leftrightarrow y)$ and $d_{n}=\gamma_{E P o s}(x \wedge y)$. Then $e_{j} \cap\left(d_{n-1} \cap f_{n-1}\right) \subseteq d_{n}$ for $e_{1}=\gamma_{E P o s}(x)$ and $e_{2}=\gamma_{E P o s}(y)$ but $\left(e_{1} \oplus^{\prime} e_{2}\right) \cap\left(d_{n-1} \cap f_{n-1}\right)=\gamma_{E P o s}((x \curlyvee y) \wedge 1 \wedge(x \Leftrightarrow y))=$ $\gamma_{E P o s}(x \Leftrightarrow y) \nsubseteq \gamma_{E P o s}(x \wedge y)=d_{n}$. Thus there is no unique $e$ maximising precision.

\section{Example 5.2}

Identity $\rho_{\mathrm{id}}=\lambda x . x$ is the trivial semi-morphism between $\left\langle\wp^{\downarrow}(\mathcal{C}), \subseteq, \cup, \cap, \mathcal{C}, \emptyset, \exists, d\right\rangle$ and $\left\langle\wp^{\downarrow}(\mathcal{C}), \subseteq, \cup, \cap, \mathcal{C}, \emptyset, \exists, d\right\rangle$ where the pseudo-complement is given by $C_{1} \rightarrow^{\prime} C_{2}=$ $\left\{c \in \mathcal{C} \mid \forall c^{\prime} \unlhd c . c^{\prime} \in C_{1} \Rightarrow c^{\prime} \in C_{2}\right\}$ (Birkhoff, 1967).

\section{Example 5.3}

Recall that $\rho_{P o s}$ is a semi-morphism between $\left\langle\wp^{\downarrow}(E q n), \subseteq, \cup, \cap, E q n, \emptyset, \exists, d\right\rangle$ and $\left\langle\rho_{\text {Pos }}\left(\wp^{\downarrow}(E q n)\right), \subseteq, \oplus^{\prime}, \cap, E q n, \rho_{\text {Pos }}(\emptyset), \exists^{\prime}, d^{\prime}\right\rangle$. Although $\oplus^{\prime} \neq \cup, \rho_{\text {Pos }}\left(\wp^{\downarrow}(E q n)\right)$ is a sub-cHa of $\wp^{\downarrow}(E q n)$ with respect to $\cap$ and $\rightarrow^{\prime}$ (Scozzari, to appear). Moreover, pseudo-complement (intuitionistic implication) $\rightarrow^{\prime}$ coincides with classic implication $\Rightarrow$ in the sense that $C_{1} \rightarrow^{\prime} C_{2}=\gamma_{P o s}\left(f_{1} \Rightarrow f_{2}\right)$ where $C_{i}=\gamma_{\text {Pos }}\left(f_{i}\right)$. This follows since $V \models f_{2} \models\left(\neg f_{1}\right) \vee f_{2}$ and thus $f_{1} \Rightarrow f_{2} \in$ Pos. Moreover, $f_{1} \wedge f \models f_{2}$ iff $\models\left(f_{1} \wedge f\right) \Rightarrow f_{2}$ iff $\models f \Rightarrow\left(\neg f_{1}\right) \vee f_{2}$ iff $f \models\left(\neg f_{1}\right) \vee f_{2}$. Hence $C_{1} \rightarrow^{\prime} C_{2}=\oplus^{\prime}\left\{C \in \rho_{\text {Pos }}\left(\wp^{\downarrow}(E q n)\right) \mid C_{1} \cap C \subseteq C_{2}\right\}=\gamma_{\text {Pos }}\left(f_{1} \Rightarrow f_{2}\right)$. Thus $\rightarrow^{\prime}$ is finitely computable for $\rho_{P o s}$. Finally note that $\neg$ and $\vee$ are defined on $B o o l$ rather than Pos since $\neg f \notin$ Pos iff $f \in$ Pos.

\section{Example 5.4}

Now consider the problem of inferring an initial $c$ for $\left\langle p_{n-1}\left(\vec{x}_{n-1}\right), p_{n}\left(\vec{x}_{n}\right) ; c\right\rangle$ within $\rho_{\text {Pos }}\left(\wp^{\downarrow}(E q n)\right)$. Analogous to example 5.1, let $d_{n-1}=\gamma_{\text {Pos }}(1), f_{n-1}=\gamma_{\text {Pos }}(x \Leftrightarrow y)$ and $d_{n}=\gamma_{P o s}(x \wedge y)$. Then $e_{j} \cap\left(d_{n-1} \cap f_{n-1}\right) \subseteq d_{n}$ for $e_{1}=\gamma_{P o s}(x)$ and $e_{2}=$ $\gamma_{\text {Pos }}(y)$ and $\left(e_{1} \oplus^{\prime} e_{2}\right) \cap\left(d_{n-1} \cap f_{n-1}\right)=\gamma_{\text {Pos }}((x \vee y) \wedge 1 \wedge(x \Leftrightarrow y))=\gamma_{\text {Pos }}(x \wedge y)=d_{n}$. Thus there is a unique $e$ maximising precision.

Since $\left\langle\rho\left(\wp^{\downarrow}(\mathcal{C})\right), \subseteq, \oplus^{\prime}, \cap, \mathcal{C}, \rho(\emptyset), \exists^{\prime}, d^{\prime}\right\rangle$ is a cylindric constraint system, it follows 
that $e \subseteq \exists_{x}^{\prime}(e)$ for all $e \in \rho(\wp \downarrow(\mathcal{C}))$. A consequence of $e \subseteq \exists_{x}^{\prime}(e)$ is that projection approximates from above. Approximation from above, however, is not entirely appropriate for backward analysis. In particular, observe that if $\langle g ; c\rangle \nRightarrow_{P}^{\star} \diamond$ for all $c \in e$, then it does not necessarily follow that $\langle g ; c\rangle \nexists_{P}^{\star} \diamond$ for all $c \in \exists_{x}^{\prime}(e)$. What is required is a dual notion of projection, say denoted $\forall^{\prime}$, that approximates from below. Then $\langle g ; c\rangle \nRightarrow{ }_{P}^{\star} \diamond$ for all $c \in \forall_{x}^{\prime}(e)$. Although $\forall^{\prime}$ is an abstract operator, the concept is defined for an arbitrary cylindric constraint system for generality.

\section{Definition 5.1}

If $\langle\mathcal{C}, \unlhd, \oplus, \otimes, 1,0, \exists, d\rangle$ is a cylindric constraint system and $x \in V$ then $\forall_{x}: \mathcal{C} \rightarrow \mathcal{C}$ is a monotonic operator such that: $\exists_{x}\left(\forall_{x}(c)\right) \unlhd c$ and $c \unlhd \forall_{x}\left(\exists_{x}(c)\right)$ for all $c \in \mathcal{C}$.

Recall that $\exists_{x}$ is monotonic and thus $\alpha$ is the lower adjoint of $\gamma$ and $\gamma$ is the upper adjoint of $\alpha$. More exactly, it follows that $\forall_{x}$ can be automatically constructed from $\exists_{x}$ by $\forall_{x}(c)=\oplus\left\{c^{\prime} \in \mathcal{C} \mid \exists_{x}\left(c^{\prime}\right) \unlhd c\right\}$. Observe that this ensures that $\forall_{x}$ is the most precise projection operator from below. For succinctness, define $\forall_{\left\{x_{1}, \ldots, x_{n}\right\}}(c)=$ $\forall_{x_{1}}\left(\ldots\left(\forall_{x_{n}}(c)\right)\right)$ and $\bar{\forall}_{X}(c)=\forall_{F V(c) \backslash X}(c)$.

\section{Example 5.5}

For $\rho_{\text {id }}$, let $\forall_{x}^{\prime}(C)=\downarrow\left(\left\{c \in C \mid \exists_{x}(c)=c\right\}\right)$.

\section{Example 5.6}

For $\rho_{P o s}$, let $\forall_{x}^{\prime}(C)=\gamma_{P o s}\left(f^{\prime}\right)$ if $f^{\prime} \in$ Pos otherwise $\forall_{x}^{\prime}(C)=\gamma_{P o s}(0)$ where $C=$ $\gamma_{P o s}(f)$ and $f^{\prime}=f[x \mapsto 0] \wedge f[x \mapsto 1]$. Observe that $\exists_{x}(f)[x \mapsto 0] \wedge \exists_{x}(f)[x \mapsto 1]=$ $\exists_{x}(f)$ and hence $C \subseteq \exists_{x}^{\prime}(C)=\forall_{x}^{\prime}\left(\exists_{x}^{\prime}(C)\right)$ as required. Moreover, if $\forall_{x}^{\prime}(C)=\gamma_{P o s}(0)$ then $\exists_{x}^{\prime}\left(\forall_{x}^{\prime}(C)\right)=\gamma_{P o s}(0) \subseteq C$. Otherwise $\exists_{x}(f[x \mapsto 0] \wedge f[x \mapsto 1])=f[x \mapsto$ $0] \wedge f[x \mapsto 1] \models f$. Thus $\exists_{x}^{\prime}\left(\forall_{x}^{\prime}(C)\right) \subseteq C$ as required. Finally, note that $\forall_{x}^{\prime}$ is finitely computable for $\rho_{P o s}$. For example if $C_{i}=\gamma_{P o s}\left(f_{i}\right), f_{1}=(x \Leftarrow y), f_{2}=(x \wedge y)$ and $f_{3}=(x \vee y)$, then $\forall_{x}^{\prime}\left(C_{i}\right)=\gamma_{P o s}\left(f_{i}^{\prime}\right)$ where $f_{1}^{\prime}=0, f_{2}^{\prime}=0$ and $f_{3}^{\prime}=y$.

Backward analysis can now be formalised as follows.

Definition 5.2

Given a constraint logic program $P$ over a semi-cylindric constraint system $\mathcal{C}$ with assertions over $\rho\left(\wp^{\downarrow}(\mathcal{C})\right)$, the operator $\mathcal{D}_{P}^{\rho, \mathcal{C}}: \operatorname{Int} t^{\operatorname{cod}(\rho)} \rightarrow \operatorname{In} t^{\operatorname{cod}(\rho)}$ is defined by:

$$
\mathcal{D}_{P}^{\rho, \mathcal{C}}(D)=\bigcup\left\{E \mid \begin{array}{ccc}
\forall & {[p(\vec{x}):-e] \approx \in E} & \cdot \\
\forall & p(\vec{x}):-C \diamond c, p_{1}\left(\vec{x}_{1}\right), \ldots, p_{n}\left(\vec{x}_{n}\right) \in P & \cdot \\
\exists & \left\{\left[p_{i}\left(\vec{x}_{i}\right):-f_{i}\right]_{\approx}\right\}_{i=1}^{n} \subseteq F & \cdot \\
\exists & \left\{\left[p_{i}\left(\vec{x}_{i}\right):-d_{i}\right]_{\approx}\right\}_{i=1}^{n} \subseteq D & \cdot \\
& e_{n+1}=\mathcal{C} \wedge e_{i}=d_{i} \cap\left(f_{i} \rightarrow^{\prime} e_{i+1}\right) & \wedge \\
& e \subseteq \bar{\forall}_{\vec{x}}^{\prime}\left(e_{0}\right) \wedge e_{0}=C \cap\left(\rho(\downarrow(c)) \rightarrow^{\prime} e_{1}\right)
\end{array}\right\}
$$

where $[F]_{\equiv}=\mathcal{F}^{\operatorname{cod}(\rho)}(\rho(\downarrow(\operatorname{CLP}(P))))$.

Since $\mathcal{D}$ is parameterised by $\rho$ and $\mathcal{C}$ it can interpreted as a backward analysis framework. $\mathcal{D}$ requires $F$, the success patterns of the program obtained by discarding the assertions, to be pre-computed. $\mathcal{D}$ considers each clause in the program in turn and calculates those states which ensure that the clause (and those it calls) will not violate an assertion. An abstraction which characterises these states is calculated by 
propagating requirements, represented as abstractions, right-to-left by repeated application of pseudo-complement. Projection from below then computes those states which, when restricted to the head variables, still ensure that no error arises in the clause (and those it calls). Repeated application of $\mathcal{D}$ yields a decreasing sequence of interpretations.

The operator $\mathcal{D}_{P}^{\rho, \mathcal{C}}$ lifts to $\operatorname{Int} t^{\operatorname{cod}(\rho)} / \equiv$ by $\mathcal{D}_{P}^{\rho, \mathcal{C}}\left([D]_{\equiv)}\right)=\left[\mathcal{D}_{P}^{\rho, \mathcal{C}}(D)\right]_{\equiv}$. Since $\langle\operatorname{cod}(\rho), \sqsubseteq, \sqcup, \Pi\rangle$ is a complete lattice, $\mathcal{D}_{P}^{\rho, \mathcal{C}}$ will possess a gfp if $\mathcal{D}_{P}^{\rho, \mathcal{C}}$ is monotonic. The existence of $\operatorname{gfp}\left(\mathcal{D}_{P}^{\rho, \mathcal{C}}\right)$ is guaranteed by the following result since co-continuity implies monotonicity.

Proposition 5.1

$\mathcal{D}_{P}^{\rho, \mathcal{C}}:$ Int $t^{\operatorname{cod}(\rho)} / \equiv \rightarrow$ Int $t^{\operatorname{cod}(\rho)} / \equiv$ is co-continuous.

Since $\operatorname{gfp}\left(\mathcal{D}_{P}^{\rho, \mathcal{C}}\right)$ exists, a backward fixpoint semantics can be defined $\mathcal{D}^{\rho, \mathcal{C}}(P)=\operatorname{gfp}\left(\mathcal{D}_{P}^{\rho, \mathcal{C}}\right)$ and computed by lower Kleene iteration. To establish a connection between $\mathcal{D}^{\rho, \mathcal{C}}(P)$ and the operational semantics of $P$, it is useful to annotate the goals of a state with their depth in the computation tree. To formalise this idea $\Rightarrow_{P}$ is lifted to the annotated states $\operatorname{Con} f_{\diamond}=C o n f \cup\{\diamond\}$ where Conf $=$ Goal $\times \mathcal{C} \times \mathbb{N}^{\star}$ to obtain the transition system $\Rightarrow_{P}$.

\section{Definition 5.3}

Given a constraint logic program with assertions $P$ over a semi-cylindric constraint system $\mathcal{C}, \Rightarrow_{P} \subseteq$ Conf $\times C o n f_{\diamond}$ is the least relation such that:

$$
\langle p(\vec{x}), g ; c ; n \cdot h\rangle \Rightarrow_{P} \begin{cases}\diamond & \text { if }\langle p(\vec{x}), g ; c\rangle \Rightarrow_{P} \diamond \\ \left\langle g^{\prime}, g ; c^{\prime} ;(n+1)^{\left|g^{\prime}\right|} \cdot h\right\rangle & \text { if }\langle p(\vec{x}), g ; c\rangle \Rightarrow_{P}\left\langle g^{\prime}, g ; c^{\prime}\right\rangle\end{cases}
$$

The sequence $(n+1)^{\left|g^{\prime}\right|}$ denotes $\left|g^{\prime}\right|$ concatenations of $n+1$. The following result relates the depth of the goals of the annotated states to the iterates obtained by lower Kleene iteration. Informally, it says that if a constrained atom $p(\vec{x}):-e$ occurs in the interpretation obtained by applying $\mathcal{D} k$ times, and $e$ characterises an initial state (in a certain sense), and the depth of the goals in a derivation starting at the initial state does not exceed $k$, then the derivation will not violate an assertion. The main safety theorem flows out of this result.

\section{Lemma 5.1}

Let $\left\langle p(\vec{y}) ; c^{\prime \prime} ; 1\right\rangle=s_{1} \Rightarrow_{P}^{\star} s_{n} \Rightarrow_{P} \diamond, s_{i}=\left\langle g_{i} ; c_{i} ; h_{i}\right\rangle$ and $\left(\mathcal{D}_{P}^{\rho, \mathcal{C}}\right)^{k}(\top)=\left[D_{k}\right]_{\equiv}$. If $\max \left(\left\{\max \left(h_{i}\right) \mid 1 \leq i \leq n\right\}\right) \leq k$ and $[p(\vec{y}):-e]_{\approx} \in D_{k}$ then $\bar{\exists}_{\vec{y}}\left(c^{\prime \prime}\right) \notin \bar{\exists}_{\vec{y}}(e)$.

Theorem 5.1

If $\mathcal{D}^{\rho, \mathcal{C}}(P)=[D]_{\equiv},[p(\vec{y}):-e]_{\approx} \in D$ and $c \in \bar{\exists}_{\vec{y}}(e)$ then $\langle p(\vec{y}) ; c\rangle \nRightarrow_{P}^{\star} \diamond$.

\section{Experimental evaluation}

In order to evaluate the usefulness of the analysis framework presented in section 5 , a backward Pos analyser has been constructed for inferring calling modes. The fixpoint component of the analyser is coded in SICStus Prolog 3.8.3. The domain operations are coded in $\mathrm{C}$ and are essentially the binary decision diagram (BDD) 
routines written by Armstrong and Schachte (Armstrong et al., 1998). The analyser takes, as input, a program written in a declarative subset of ISO Prolog. It outputs a mode for each program predicate. The safety result of theorem 5.1 ensures that if a call to a predicate is at least as instantiated as the inferred mode, then the call will not violate an instantiation requirement. Modes are expressed as grounding dependencies (Armstrong et al., 1998).

The implementation follows the framework defined in section 5 very closely. The analyser was straightforward to implement as it is essentially two bottom-up fixpoint computations: one for $\mathcal{F}$ and the other for $\mathcal{D}$. The only subtlety is in handling the builtins. For each builtin, it is necessary to select a grounding dependency that is sufficient for avoiding an instantiation error. This is an lower approximation (the required mode of table 1). It is also necessary to specify behaviour on success. This is an upper-approximation (the success mode of table 1 ). The lower approximations are the assertions that are added to Prolog program to obtain a constraint logic program with assertions.

Interestingly, the success mode does not always entail the required mode. Univ (=..) illustrates this. A sufficient but not necessary condition for univ not to error is that either the first or second argument is ground. This cannot be weakened in Pos (but could be weakened in a type dependency domain (Codish \& Lagoon, 2000) that expressed rigid lists). The success mode is that the first argument is ground iff the second argument is ground (which does not entail the required mode). Note too that keysort and sort error if their first argument is free. A sufficient mode for expressing this requirement is that the first argument is ground. Again, this requirement cannot be weakened in Pos.

The analyser has been applied to some standard Prolog benchmarks which can be found at http://www.oakland.edu/ $12 \mathrm{lu} /$ benchmarks-BG.zip. The results of the analysis, that is, the calling modes for the predicates in the smaller benchmarks, are given in table 2 . The results, though surprising in some cases (see sort of permSort and insert of treesort for example) have been verified by hand and appear to be optimal for Pos. The analysis, of course, can be applied to larger programs (though it becomes very difficult to verify the results by hand) and table 3 demonstrates that the analysis scales smoothly to medium-scale programs at least. The table lists the larger benchmarks (which possibly include some unreachable code) in terms of increasing size measured by the total number of atoms in the source. The abs column records the time in milliseconds required to read, parse and normalise the source into the ground program representation used by the analyser; lfp is the time needed to compute the fixpoint characterising the success modes; $g f p$ is the time needed to compute the calling modes; and finally sum is the total analysis time. This includes the (usually negligible) overhead of annotating the source with the modes required by builtins. Timings were performed on a Dell GX200 1GHz PC with $128 \mathrm{MB}$ memory running Windows 2000 . The timings suggest that the analysis is practical at least for medium-scale programs (though the running time for BDDs can be sensitive to the particular dependencies that arise). Moreover, with a stateof-the-art GER factorised BDD package (Bagnara \& Schachte, 1999) the analysis would be faster. Interestingly, the time to compute the lfp often dominates the 


\begin{tabular}{|c|c|c|}
\hline builtin & required mode & success mode \\
\hline $\begin{array}{l}t_{1}==t_{2}, t_{1} \backslash==t_{2}, \quad t_{1} @<t_{2}, t_{1} @>t_{2}, \\
t_{1} @=<t_{2}, \quad t_{1} @>=t_{2}, \quad t_{1} \backslash=t_{2}, \quad !, \\
\operatorname{compound}\left(t_{1}\right), \operatorname{display}\left(t_{1}\right), \operatorname{listing}, \operatorname{listing}\left(t_{1}\right), \\
\text { nl, nonvar }\left(t_{1}\right), \operatorname{print}\left(t_{1}\right), \operatorname{portray} \operatorname{clause}\left(t_{1}\right), \\
\operatorname{read}\left(t_{1}\right), \operatorname{repeat,} \operatorname{true}, \operatorname{var}\left(t_{1}\right), \operatorname{write}\left(t_{1}\right), \\
\text { writeq }\left(t_{1}\right)\end{array}$ & true & true \\
\hline $\begin{array}{l}\text { atom }\left(t_{1}\right), \quad \text { atomic }\left(t_{1}\right), \quad \text { compare }\left(t_{1}, t_{2}, t_{3}\right), \\
\text { float }\left(t_{1}\right), \text { ground }\left(t_{1}\right), \text { integer }\left(t_{1}\right), \text { number }\left(t_{1}\right)\end{array}$ & true & $f_{1}$ \\
\hline $\operatorname{length}\left(t_{1}, t_{2}\right)$ & true & $f_{2}$ \\
\hline statistics $\left(t_{1}, t_{2}\right)$ & true & $g_{1}$ \\
\hline abort, fail, false & true & false \\
\hline $\operatorname{keysort}\left(t_{1}, t_{2}\right), \operatorname{sort}\left(t_{1}, t_{2}\right)$ & $f_{1}$ & $g_{2}$ \\
\hline $\operatorname{tab}\left(t_{1}\right), \operatorname{put}\left(t_{1}\right)$ & $f_{1}$ & $f_{1}$ \\
\hline$t_{1}$ is $t_{2}$ & $f_{2}$ & $g_{1}$ \\
\hline $\begin{array}{l}t_{1}=:=t_{2}, \quad t_{1}=\backslash=t_{2}, \quad t_{1}<t_{2}, \quad t_{1}>t_{2} \\
t_{1}=<t_{2}, t_{1}>=t_{2}\end{array}$ & $g_{1}$ & $g_{1}$ \\
\hline $\arg \left(t_{1}, t_{2}, t_{3}\right)$ & $g_{1}$ & $g_{3}$ \\
\hline name $\left(t_{1}, t_{2}\right)$ & $g_{4}$ & $g_{1}$ \\
\hline$t_{1}=. . t_{2}$ & $g_{4}$ & $g_{2}$ \\
\hline functor $\left(t_{1}, t_{2}, t_{3}\right)$ & $g_{5}$ & $g_{6}$ \\
\hline
\end{tabular}

Table 1. Abstracting builtins where $f_{i}=\wedge \operatorname{var}\left(t_{i}\right), g_{1}=f_{1} \wedge f_{2}, g_{2}=f_{1} \Leftrightarrow f_{2}$, $g_{3}=f_{1} \wedge\left(f_{2} \Rightarrow f_{3}\right), g_{4}=f_{1} \vee f_{2}, g_{5}=f_{1} \vee\left(f_{2} \wedge f_{3}\right)$ and $g_{6}=f_{2} \wedge f_{3}$.

whole analysis. BDD widening will be required to analyse very large applications but this is a study within itself (Heaton et al., 2000).

\section{Related work}

Our work was motivated by the recent revival of interest in logic programming with assertions (Boye et al., 1997; Puebla et al., 2000a). For example, (Puebla et al., 2000b) argues that it is useful to trap an unexpected call to a predicate with an assertion otherwise a program may error at a point that is far from the source of the problem. Moreover, (Puebla et al., 2000a) observe that predicates are normally written with an expectation on the initial calling pattern, and hence provide an entry assertion to make the, moding say, of the top-level queries explicit. Our work shows how entry assertions can be automatically synthesised which ensure that instantiation errors do not occur while executing the program.

The most closely related work concerns the demand analysis of ccp (Debray, 1993; Falaschi et al., 2000). A demand analysis for the ccp language Janus (Saraswat et al., 1990) is proposed in (Debray, 1993) which determines whether or not a predicate is uni-modal. A predicate is uni-modal iff the argument tuple for each clause share the same minimal pattern of instantiation necessary for reduction. The demand analysis of a predicate simply traverses the head and guard of each clause to determine the extent to which arguments have to be instantiated. Body atoms need not be considered so the analysis does not involve a fixpoint computation. A related paper (Debray et al., 1992) presents a goal-dependent (forward) analysis that detects those ccp predicates which can be scheduled left-to-right without deadlock. If assertions 


\begin{tabular}{|c|c|c|}
\hline benchmark & predicate & mode \\
\hline bubblesort & $\begin{array}{l}\operatorname{sort}\left(x_{1}, x_{2}\right) \\
\operatorname{ordered}\left(x_{1}\right) \\
\operatorname{append}\left(x_{1}, x_{2}, x_{3}\right)\end{array}$ & $\begin{array}{c}x_{1} \\
x_{1} \\
\text { true }\end{array}$ \\
\hline$\overline{\operatorname{dnf}}$ & $\begin{array}{l}\text { go } \\
\operatorname{dnf}\left(x_{1}, x_{2}\right) \\
\operatorname{norm}\left(x_{1}, x_{2}\right) \\
\operatorname{literal}\left(x_{1}\right) \\
\end{array}$ & $\begin{array}{l}\text { true } \\
\text { true } \\
\text { true } \\
\text { true }\end{array}$ \\
\hline heapify & $\begin{array}{l}\operatorname{greater}\left(x_{1}, x_{2}\right) \\
\operatorname{adjust}\left(x_{1}, x_{2}, x_{3}, x_{4}\right) \\
\operatorname{heapify}\left(x_{1}, x_{2}\right)\end{array}$ & $\begin{array}{c}x_{1} \wedge x_{2} \\
\left(\begin{array}{c}\left(x_{1} \wedge x_{4}\right) \\
\left(x_{1} \wedge x_{2} \wedge x_{3}\right) \\
\left(\neg x_{2} \wedge \neg x_{3} \wedge x_{4}\right)\end{array}\right) \\
x_{1}\end{array}$ \\
\hline permSort & $\begin{array}{l}\operatorname{select}\left(x_{1}, x_{2}, x_{3}\right) \\
\operatorname{ordered}\left(x_{1}\right) \\
\operatorname{permutation}\left(x_{1}, x_{2}\right) \\
\operatorname{sort}\left(x_{1}, x_{2}\right)\end{array}$ & $\begin{array}{c}\text { true } \\
x_{1} \\
\text { true } \\
x_{1} \vee x_{2}\end{array}$ \\
\hline queens & $\begin{array}{l}\operatorname{noattack}\left(x_{1}, x_{2}, x_{3}\right) \\
\operatorname{safe}\left(x_{1}\right) \\
\operatorname{delete}\left(x_{1}, x_{2}, x_{3}\right) \\
\operatorname{perm}\left(x_{1}, x_{2}\right) \\
\text { queens }\left(x_{1}, x_{2}\right)\end{array}$ & $\begin{array}{c}x_{1} \wedge x_{2} \wedge x_{3} \\
x_{1} \\
\text { true } \\
\text { true } \\
x_{1} \vee x_{2}\end{array}$ \\
\hline quicksort & $\begin{array}{l}\operatorname{append}\left(x_{1}, x_{2}, x_{3}\right) \\
\operatorname{qsort}\left(x_{1}, x_{2}\right) \\
\operatorname{partition}\left(x_{1}, x_{2}, x_{3}, x_{4}\right)\end{array}$ & $\begin{array}{c}\text { true } \\
x_{1} \\
x_{2} \wedge\left(x_{1} \vee\left(x_{3} \wedge x_{4}\right)\right)\end{array}$ \\
\hline treeorder & $\begin{array}{l}\operatorname{member}\left(x_{1}, x_{2}\right) \\
\operatorname{select}\left(x_{1}, x_{2}, x_{3}\right) \\
\operatorname{split}\left(x_{1}, x_{2}, x_{3}, x_{4}\right) \\
\operatorname{split}\left(x_{1}, \ldots, x_{7}\right) \\
\operatorname{visits} 2 \operatorname{tree}\left(x_{1}, x_{2}, x_{3}\right) \\
\operatorname{v2t}\left(x_{1}, x_{2}, x_{3}\right)\end{array}$ & $\begin{array}{l}\text { true } \\
\text { true } \\
\text { true } \\
\text { true } \\
\text { true } \\
\text { true }\end{array}$ \\
\hline treesort & $\begin{array}{l}\text { tree_to_list_aux }\left(x_{1}, x_{2}, x_{3}\right) \\
\text { tree_to_list }\left(x_{1}, x_{2}\right) \\
\text { list_to_tree }\left(x_{1}, x_{2}\right) \\
\operatorname{insert} l i s t\left(x_{1}, x_{2}, x_{3}\right) \\
\operatorname{insert}\left(x_{1}, x_{2}, x_{3}\right) \\
\operatorname{treesort}\left(x_{1}, x_{2}\right)\end{array}$ & $\begin{array}{c}\text { true } \\
\text { true } \\
x_{1} \\
x_{1} \wedge x_{2} \\
x_{1} \wedge\left(x_{2} \vee x_{3}\right) \\
x_{1}\end{array}$ \\
\hline
\end{tabular}

Table 2. Precision of the Mode Analysis (small benchmarks)

are used to approximate synchronisation, then the analysis described in this paper can be re-interpreted as a backward suspension analysis of ccp under left-to-right scheduling.

When reasoning about module interaction it can be advantageous to reverse the traditional deductive approach to abstract interpretation that is based on the abstract unfolding of abstract goals. In particular (Giacobazzi, 1998) shows how abduction and abstraction can be combined to compute those properties that one module must satisfy to ensure that its composition with another fulfils certain requirements. Abductive analysis can, for example, determine how an optimisation in one module depends on a predicate defined in another module. Abductive analysis is related to the backward analysis presented in this paper since abduction is the 


\begin{tabular}{r|r|rrrrr|r|rrrr} 
file & size & abs & lfp & gfp & sum & file & size & abs & lfp & gfp & sum \\
\hline astar & 100 & 10 & 10 & 0 & 20 & tictactoe & 258 & 20 & 10 & 10 & 40 \\
fft & 104 & 20 & 0 & 10 & 30 & jons2 & 261 & 20 & 10 & 0 & 30 \\
knight & 105 & 10 & 0 & 0 & 10 & kalah & 269 & 30 & 10 & 20 & 60 \\
browse_wamcc & 106 & 10 & 0 & 0 & 10 & draw & 289 & 70 & 91 & 40 & 201 \\
cal_wamcc & 108 & 10 & 10 & 0 & 20 & cs_r & 311 & 40 & 20 & 10 & 70 \\
life & 110 & 10 & 10 & 10 & 30 & reducer & 320 & 40 & 30 & 0 & 70 \\
crypt_wamcc & 113 & 10 & 0 & 0 & 10 & sdda & 336 & 20 & 21 & 0 & 41 \\
cry_mult & 118 & 10 & 10 & 10 & 30 & bryant & 349 & 30 & 120 & 21 & 171 \\
browse & 125 & 10 & 10 & 0 & 20 & ga & 363 & 50 & 30 & 20 & 100 \\
bid & 128 & 10 & 10 & 0 & 20 & neural & 378 & 30 & 10 & 0 & 40 \\
disj_r & 148 & 30 & 0 & 10 & 40 & press & 381 & 30 & 20 & 0 & 50 \\
consultant & 151 & 20 & 0 & 10 & 30 & peep & 414 & 50 & 20 & 10 & 80 \\
ncDP & 156 & 10 & 10 & 0 & 20 & nbody & 421 & 40 & 20 & 20 & 80 \\
tsp & 162 & 30 & 20 & 10 & 60 & eliza & 432 & 50 & 20 & 0 & 70 \\
elex_scanner & 165 & 20 & 10 & 0 & 30 & read & 434 & 40 & 20 & 10 & 70 \\
robot & 165 & 10 & 10 & 0 & 20 & simple_analyzer & 512 & 90 & 701 & 20 & 811 \\
sorts & 172 & 0 & 10 & 10 & 20 & ann & 547 & 50 & 30 & 10 & 90 \\
cs2 & 175 & 30 & 10 & 10 & 50 & diffsimpsv & 681 & 61 & 100 & 0 & 161 \\
scc & 175 & 10 & 141 & 0 & 151 & arch1 & 692 & 50 & 40 & 10 & 100 \\
bp0-6 & 201 & 20 & 10 & 0 & 30 & asm & 800 & 60 & 40 & 30 & 130 \\
bnet & 205 & 20 & 20 & 0 & 40 & poker & 962 & 81 & 70 & 10 & 161 \\
jons & 222 & 40 & 0 & 10 & 50 & pentomino & 981 & 50 & 40 & 80 & 170 \\
mathlib & 226 & 10 & 10 & 0 & 20 & chat & 1037 & 411 & 1422 & 1082 & 2915 \\
intervals & 230 & 20 & 10 & 10 & 40 & sim_v5-2 & 1308 & 80 & 70 & 0 & 150 \\
barnes_hut & 240 & 40 & 30 & 40 & 110 & semigroup & 2328 & 180 & 90 & 60 & 350
\end{tabular}

Table 3. Speed of the Mode Analysis (medium-scale benchmarks)

inverse image of a forward semantics whereas pseudo-complement is the inverse image of conjunction - the basic computational step in forward (and backward) semantics.

The termination inference engine of (Genaim \& Codish, 2001) decomposes the cTI analyser of (Mesnard, 1996) into two components: a termination checker (Codish \& Taboch, 1999) and the backward analysis described in this paper. First, the termination inference engine computes a set of binary clauses which describe possible loops in the program with size relations. Second, a Boolean function is inferred for each predicate that describes moding conditions sufficient for each loop to only be executed a finite number of times. Third, the backward analysis described in this paper is applied to infer initial modes by calculating a greatest fixpoint which guarantee that the moding conditions hold and thereby assure termination. Interestingly, the cTI analyser involves a $\mu$-calculus solver to compute the greatest fixpoint of an equivalent (though more complex) system of equations. This seems to suggest that greatest fixpoints are important in backward analysis.

Cousot and Cousot (Cousot \& Cousot, 1992) explain how a backward collecting semantics can be deployed to precisely characterise states that arise in finite SLD-derivations. First, they present a forward collecting semantics that records the descendant states that arise from a set of initial states. Second, they present a dual (backward) collecting semantics that records those states which occur as ascendant 
states of the final states. By combining both semantics, they characterise the set of descendant states of the initial states which are also ascendant states of the final states of the transition system. This use of backward analysis is primarily as a device to improve the precision of a classic goal-dependent analysis. Our work is more radical in the sense that it shows how a bottom-up analysis performed in a backward fashion, can be used to characterise initial queries. Moreover it is used for lower approximation rather than upper approximation.

Mazur, Janssens and Bruynooghe (Mazur et al., 2000) present a kind of ad hoc backward analysis to derive reuse conditions from a goal-independent reuse analysis for Mercury (Somogyi et al., 1996). The analysis propagates reuse information from a point where a structure is decomposed in a clause to the point where the clause is invoked in its parent clause. This is similar in spirit to how demand is passed from a callee to a caller in the backward analysis described in this paper. However, the reuse analysis does not propagate information right-to-left across a clause using pseudo-complement, and so one interesting topic for future work will to be relate these two analyses. Another matter for future work, will be to investigate the extent to which our backward mode analysis can be reconstructed by inverting abstract functions (Hughes \& Launchbury, 1994).

\section{Conclusion}

We have shown how abstract interpretation, and specifically a backward analysis, can infer moding properties which if satisfied by the initial query, come with the guarantee that the program and query cannot generate instantiation errors. Backward analysis has other applications in termination inference and also in inferring queries for which the builtins called from within the program behave predictably in the presence of rational trees. The analysis is composed of two bottom-up fixpoint calculations, a lfp and a gfp, both of which are straightforward to implement. The Ifp characterises success patterns. The gfp, uses these success patterns to infer safe initial calling patterns. It propagates moding requirements right-to-left, against the control-flow, using the pseudo-complement operator. This operator fits with backward analysis since it enables moding requirements to be minimised (maximally weakened) in right-to-left propagation. This operator, however, requires that the computational domain be closed under Heyting completion (or equivalently condense). This requirement seems reasonable because disjunctive dependencies occur frequently in right-to-left propagation and therefore significant precision would be lost if the requirement were relaxed. Experimental evaluation has demonstrated that the analysis is practical in the sense that it can infer calling modes for mediumscaled programs. Finally, our work adds weight to the belief that condensing is an important property in the analysis of logic programs.

\section{Acknowledgements}

We thank Maurice Bruynooghe, Mike Codish, Samir Genaim, Roberto Giacobazzi, Jacob Howe, Fred Mesnard, Germán Puebla and Francesca Scozzari for helpful dis- 
cussions. We would also like to thank the anonymous referees for their comments and Peter Schachte for his BDD analyser. We also thank Roberto Bagnara for the use of some of the CHINA benchmarks. This work was supported, in part, by EPSRC grant GR/MO8769.

\section{References}

Armstrong, T., Marriott, K., Schachte, P., \& Søndergaard, H. (1998). Two Classes of Boolean Functions for Dependency Analysis. Science of Computer Programming, 31(1), $3-45$.

Bagnara, R., \& Schachte, P. (1999). Factorizing Equivalent Variable Pairs in ROBDDBased Implementations of Pos. Pages 471-485 of: International Conference on Algebraic Methodology and Software Technology. Lecture Notes in Computer Science, vol. 1548. Springer-Verlag.

Bagnara, R., Zaffanella, E., Gori, R., \& Hill, P. M. (2001). Boolean Functions for FiniteTree Dependencies. Pages 575-589 of: International Conference on Logic for Programming, Artificial Intelligence and Reasoning. Lecture Notes in Artificial Intelligence, vol. 2250. Springer-Verlag.

Birkhoff, G. (1967). Lattice Theory. AMS Press.

Bossi, A., Gabbrielli, M., Levi, G., \& Martelli, M. (1994). The s-Semantics Approach: Theory and Applications. The Journal of Logic Programming, 19/20, 149-197.

Boye, J., Drabent, W., \& Małuszyński, J. (1997). Declarative Diagnosis of Constraint Programs: an Assertion-based Approach. Pages 123-141 of: Proceedings of the Third International Workshop on Automated Debugging. University of Linköping Press.

Codish, M., \& Lagoon, V. (2000). Type Dependencies for Logic Programs using ACIunification. Theoretical Computer Science, 238, 131-159.

Codish, M., \& Taboch, C. (1999). A Semantic Basis for the Termination Analysis of Logic Programs. The Journal of Logic Programming, 41(1), 103-123.

Cousot, P., \& Cousot, R. (1982). Inductive Principles for Proving Invariance Properties of Programs. Pages 75-119 of: Tools and Notions for Program Construction. Cambridge University Press.

Cousot, P., \& Cousot, R. (1992). Abstract Interpretation and Application to Logic Programs. The Journal of Logic Programming, 13(2-3), 103-179.

Debray, S. (1993). QD-Janus: a Sequential Implementation of Janus in Prolog. Software Practice and Experience, 23(12), 1337-1360.

Debray, S., Gudeman, D., \& Bigot, P. (1992). Detection and Optimization of Suspensionfree Logic Programs. The Journal of Logic Programming, 29(1-3), 171-194.

Drabent, W., \& Małuszyński, J. (1988). Inductive Assertion Method for Logic Programs. Theoretical Computer Science, 59(1), 133-155.

Dyber, P. (1991). Inverse Image Analysis Generalises Strictness Analysis. Information and Computation, 90(2), 194-216.

Falaschi, M., Hicks, P., \& Winsborough, W. (2000). Demand Transformation Analysis for Concurrent Constraint Programs. The Journal of Logic Programming, 41(3), 185-215.

Filé, G., \& Ranzato, F. (1994). Improving Abstract Interpretations by Systematic Lifting to the Powerset. Pages 655-669 of: International Logic Programming Symposium. MIT Press.

Genaim, S., \& Codish, M. (2001). Inferring Termination Conditions for Logic Programs using Backwards Analysis. Pages 681-690 of: International Conference on Logic for 
Programming, Artificial Intelligence and Reasoning. Lecture Notes in Artificial Intelligence, vol. 2250. Springer-Verlag.

Giacobazzi, R. (1998). Abductive Analysis of Modular Logic Programs. Journal of Logic and Computation, 8(4), 457-484.

Giacobazzi, R., \& Scozzari, F. (1998). A Logical Model for Relational Abstract Domains. ACM Transactions on Programming Languages and Systems, 20(5), 1067-1109.

Giacobazzi, R., Debray, S., \& Levi, G. (1995). Generalized Semantics and Abstract Interpretation for Constraint Logic Programs. The Journal of Logic Programming, 25(3), 191-248.

Giacobazzi, R., Ranzato, F., \& Scozzari, F. (1998). Building Complete Abstract Interpretations in a Linear Logic-based Setting. Pages 215-229 of: Static Analysis Symposium. Lecture Notes in Computer Science, vol. 1503. Springer-Verlag.

Hall, C., \& Wise, D. (1989). Generating Function Versions with Rational Strictness Patterns. Science of Computer Programming, 12, 39-74.

Heaton, A., Abo-Zaed, M., Codish, M., \& King, A. (2000). A Simple Polynomial Groundness Analysis for Logic Programs. The Journal of Logic Programming, 45(1-3), 143-156.

Hughes, R. J. M., \& Launchbury, J. (1994). Reversing Abstract Interpretations. Science of Computer Programming, 22, 307-326.

Jaffar, J., \& Maher, M. J. (1994). Constraint Logic Programming: A Survey. The Journal of Logic Programming, 19/20, 503-581.

Langen, A. (1991). Advanced Techniques for Approximating Variable Aliasing in Logic Programs. Ph.D. thesis, Computer Science Department, Los Angeles, California 900890782.

Marriott, K., \& Søndergaard, H. (1993). Precise and Efficient Groundness Analysis for Logic Programs. ACM Letters on Programming Languages and Systems, 2(4), 181-196.

Mazur, N., Janssens, G., \& Bruynooghe, M. (2000). A Module Based Analysis for Memory Reuse in Mercury. Pages 1255-1269 of: Computational Logic. Lecture Notes in Artificial Intelligence, vol. 1861.

Mesnard, F. (1996). Inferring Left-terminating Classes of Queries for Constraint Logic Programs. Pages 7-21 of: Joint International Conference and Symposium on Logic Programming. MIT Press.

Mesnard, F., \& Neumerkel, U. (2001). Applying Static Analysis Techniques for Inferring Termination Conditions of Logic Programs. Pages 93-110 of: Static Analysis Symposium. Lecture Notes in Computer Science, vol. 2126. Springer-Verlag.

Mycroft, A. (1981). Abstract Interpretation and Optimising Transformations for Applicative Programs. Ph.D. thesis, University of Edinburgh.

Puebla, G., Bueno, F., \& Hermenegildo, M. (2000a). An Assertion Language for Constraint Logic Programs. Pages 23-61 of: Analysis and Visualization Tools for Constraint Programming. Lecture Notes in Computer Science, vol. 1870. Springer-Verlag.

Puebla, G., Bueno, F., \& Hermenegildo, M. (2000b). A Generic Preprocessor for Program Validation and Debugging. Pages 63-107 of: Analysis and Visualization Tools for Constraint Programming. Lecture Notes in Computer Science, vol. 1870. Springer-Verlag.

Saraswat, V., Kahn, K., \& Levy, J. (1990). Janus: a Step Towards Distributed Constraint Programming. Pages 431-446 of: North American Conference on Logic Programming. MIT Press.

Scozzari, F. (to appear). Logical Optimality of Groundness Analysis. Theoretical Computer Science.

Somogyi, Z., Henderson, F., \& Conway, T. (1996). The execution algorithm of Mercury, 
an efficient purely declarative logic programming language. The Journal of Logic Programming, 29(1-3), 17-64.

van Dalen, D. (1997). Logic and Structure. Springer.

Wadler, P., \& Hughes, R. J. M. (1987). Projections for Strictness Analysis. Pages 385-407 of: Functional Programming and Computer Architecture. Lecture Notes in Computer Science, vol. 274. Springer-Verlag. 


\section{A Proof appendix}

Proof for proposition 3.1

Proof by induction. Let $I_{0}=\emptyset, I_{0}^{\prime}=\emptyset, I_{k+1}=\mathcal{F}_{P}^{\mathcal{C}}\left(I_{k}\right)$ and $I_{k+1}^{\prime}=\mathcal{F}_{\downarrow(P)}^{\wp^{\downarrow}(\mathcal{C})}\left(I_{k}^{\prime}\right)$. To show $\downarrow\left(I_{k}\right) \sqsubseteq I_{k}^{\prime}$ since then it follows that $\downarrow\left(\operatorname{lfp}\left(\mathcal{F}_{P}^{\mathcal{C}}\right)\right)=\downarrow\left(\sqcup_{k \in \mathbb{N}} I_{k}\right) \sqsubseteq \sqcup_{k \in \mathbb{N}} \downarrow\left(I_{k}\right) \sqsubseteq$ $\sqcup_{k \in \mathbb{N}} I_{k}^{\prime}=\operatorname{lfp}\left(\mathcal{F}_{\downarrow(P)}^{\wp^{\downarrow}(\mathcal{C})}\right)$. The base case is trivial so suppose $\downarrow\left(I_{k}\right) \sqsubseteq I_{k}^{\prime}$. Let $\left[p(\vec{x}):-c^{\prime}\right] \approx \in I_{k+1}$. Then there exists $p(\vec{x}):-c, p_{1}\left(\vec{x}_{1}\right), \ldots, p_{n}\left(\vec{x}_{n}\right) \in P$ and $\left\{\left[p_{i}\left(\vec{x}_{i}\right):-c_{i}\right]_{\approx}\right\}_{i=1}^{n} \subseteq I_{k}$ such that $c^{\prime}=c \otimes \otimes_{i=1}^{n} \bar{\exists}_{\overrightarrow{x_{i}}}\left(c_{i}\right)$. Observe that $\downarrow\left(c^{\prime}\right) \subseteq$ $\downarrow(c) \cap \cap_{i=1}^{n} \downarrow\left(\bar{\exists}_{\overrightarrow{x_{i}}}\left(c_{i}\right)\right) \subseteq \downarrow(c) \cap \cap_{i=1}^{n} \bar{\exists}_{\overrightarrow{x_{i}}}\left(\downarrow\left(c_{i}\right)\right)$. But by the inductive hypothesis, there exist $\left\{\left[p_{i}\left(\vec{x}_{i}\right):-c_{i}^{\prime}\right]_{\approx}\right\}_{i=1}^{n} \subseteq I_{k}^{\prime}$ such that $\downarrow\left(c_{i}\right) \subseteq c_{i}^{\prime}$. Hence $\left[p(\vec{x}):-c^{\prime \prime}\right]_{\approx} \in I_{k+1}^{\prime}$ such that $\downarrow\left(c^{\prime}\right) \subseteq c^{\prime \prime}$ so that $I_{k+1} \sqsubseteq I_{k+1}^{\prime}$ and the result follows.

Proof for theorem 3.3

The proof tactic is analogous to that used for proposition 3.1.

Proof for corollary 3.1

Let $\mathcal{C}$ be a semi-cylindric constraint system and $\rho \in u c o\left(\wp^{\downarrow}(\mathcal{C})\right)$ be a semi-morphism. By proposition 3.1 it follows that $\downarrow\left(\mathcal{F}^{\mathcal{C}}(P)\right) \sqsubseteq \mathcal{F}^{\wp^{\downarrow}(\mathcal{C})}(\downarrow(P))$ and hence $\rho\left(\downarrow\left(\mathcal{F}^{\mathcal{C}}(P)\right)\right) \sqsubseteq$ $\rho\left(\mathcal{F}^{\wp^{\downarrow}(\mathcal{C})}(\downarrow(P))\right)$ and by theorem $3.3 \rho\left(\mathcal{F}^{\wp^{\downarrow}(\mathcal{C})}(\downarrow(P))\right) \sqsubseteq \mathcal{F}^{\operatorname{cod}(\rho)}(\rho(\downarrow(P)))$ and so the result follows.

Proof for proposition 5.1

Let $D_{n+1} \sqsubseteq D_{n}$ for all $n \in \mathbb{N}$. Put $E_{n}=\cup\left\{D_{l} \mid l \geq n\right\}$ and $E=\cap\left\{E_{n} \mid\right.$ $n \in \mathbb{N}\}$. Since $D_{n+1} \sqsubseteq D_{n}$ observe that $E_{n} \equiv D_{n}$ for all $n \in \mathbb{N}$ and hence $\mathcal{D}_{P}^{\rho, \mathcal{C}}\left(\sqcap\left\{\left[D_{n}\right]_{\equiv} \mid n \in \mathbb{N}\right\}\right)=\mathcal{D}_{P}^{\rho, \mathcal{C}}\left(\sqcap\left\{\left[E_{n}\right]_{\equiv} \mid n \in \mathbb{N}\right\}\right)=\mathcal{D}_{P}^{\rho, \mathcal{C}}\left([E]_{\equiv}\right)=\left[\mathcal{D}_{P}^{\rho, \mathcal{C}}(E)\right]_{\equiv}=$ $\left[\cap\left\{\mathcal{D}_{P}^{\rho, \mathcal{C}}\left(E_{n}\right) \mid n \in \mathbb{N}\right\}\right]_{\equiv}=\sqcap\left\{\left[\mathcal{D}_{P}^{\rho, \mathcal{C}}\left(E_{n}\right)\right]_{\equiv} \mid n \in \mathbb{N}\right\}=\sqcap\left\{\mathcal{D}_{P}^{\rho, \mathcal{C}}\left(\left[E_{n}\right]_{\equiv}\right) \mid n \in \mathbb{N}\right\}=$ $\sqcap\left\{\mathcal{D}_{P}^{\rho, \mathcal{C}}\left(\left[D_{n}\right]_{\equiv}\right) \mid n \in \mathbb{N}\right\}$.

Proof for lemma 5.1

Proof by (double) induction. Let $\left\langle p(\vec{y}) ; c^{\prime \prime} ; 1\right\rangle=s_{1} \Rightarrow_{P}^{\star} s_{n} \Rightarrow_{P} \diamond, s_{i}=\left\langle g_{i} ; c_{i} ; h_{i}\right\rangle$ and suppose $\left(\mathcal{D}_{P}^{\rho, \mathcal{C}}\right)^{k}(\top)=\left[D_{k}\right]_{\equiv}$. The outer induction is on $k$.

base case: Suppose $\max \left(\left\{\max \left(h_{i}\right) \mid 1 \leq i \leq n\right\}\right) \leq 1$ and $[p(\vec{y}):-e]_{\approx} \in D_{1}$. Thus $s_{1} \Rightarrow_{P} \diamond$ so that $s_{1} \Rightarrow_{P} \diamond$ and hence there exists $p\left(\vec{x}^{\prime}\right):-C^{\prime} \diamond c^{\prime}, g^{\prime} \in P$ such that $\partial_{\vec{y}}^{\vec{x}^{\prime}}\left(\bar{\exists}_{\vec{y}}\left(c^{\prime \prime}\right)\right) \notin C^{\prime}$. Then $\left[p\left(\vec{x}^{\prime}\right):-e^{\prime}\right] \approx \in D_{1}$ where $\bar{\exists}_{\vec{z}}\left(d_{\vec{z}, \vec{y}} \otimes e\right)=\bar{\exists}_{\vec{z}}\left(d_{\vec{z}, \vec{x}^{\prime}} \otimes e^{\prime}\right)$ and $\operatorname{var}(\vec{z}) \cap\left(\operatorname{var}(\vec{y}) \cup F V(e) \cup \operatorname{var}\left(\vec{x}^{\prime}\right) \cup F V\left(e^{\prime}\right)\right)=\emptyset$. Observe that $e^{\prime} \subseteq \bar{\forall}_{\vec{x}^{\prime}}\left(C^{\prime}\right)$ and thus $\partial_{\vec{y}}^{\vec{x}^{\prime}}\left(\bar{\exists}_{\vec{y}}(e)\right)=\bar{\exists}_{\vec{x}^{\prime}}\left(e^{\prime}\right) \subseteq \bar{\exists}_{\vec{x}^{\prime}}\left(\bar{\forall}_{\vec{x}^{\prime}}\left(C^{\prime}\right)\right)=\bar{\forall}_{\vec{x}^{\prime}}\left(C^{\prime}\right) \subseteq C^{\prime}$. Hence $\partial_{\vec{y}}^{\vec{x}^{\prime}}\left(\bar{\exists}_{\vec{y}}\left(c^{\prime \prime}\right)\right) \notin$ $\partial_{\vec{y}}^{\vec{x}^{\prime}}\left(\bar{\exists}_{\vec{y}}(e)\right)$ so that $\bar{\exists}_{\vec{y}}\left(c^{\prime \prime}\right) \notin \bar{\exists}_{\vec{y}}(e)$ as required.

inductive case: Suppose $k=\max \left(\left\{\max \left(h_{i}\right) \mid 1 \leq i \leq n\right\}\right)>1$ and $[p(\vec{y}):-e]_{\approx} \in D_{k}$. Suppose, for the sake of a contradiction, that $\bar{\exists}_{\vec{y}}\left(c^{\prime \prime}\right) \in \bar{\exists}_{\vec{y}}(e)$. Since $k>1$ there exists $w=p(\vec{x}):-C \diamond c, p_{1}\left(\vec{x}_{1}\right), \ldots, p_{l}\left(\vec{x}_{l}\right) \in P, \varphi \in \operatorname{Ren}$ such that $\varphi(\operatorname{CLP}(w))=$ $p\left(\vec{x}^{\prime}\right):-c^{\prime}, p_{1}\left(\vec{x}_{1}^{\prime}\right), \ldots, p_{l}\left(\vec{x}_{l}^{\prime}\right) \ll_{s_{1}} \operatorname{CLP}(P)$ and $s_{2}=\left\langle p_{1}\left(\vec{x}_{1}^{\prime}\right), \ldots, p_{l}\left(\vec{x}_{l}^{\prime}\right) ; c_{1}^{\prime} ; 2^{l}\right\rangle$ and $c_{1}^{\prime}=c^{\prime \prime} \otimes d_{\vec{y}, \vec{x}^{\prime}} \otimes c^{\prime}$. Suppose $\left\langle p_{1}\left(\vec{x}_{1}^{\prime}\right) ; c_{1}^{\prime}\right\rangle \Rightarrow_{P}^{\star}\left\langle\epsilon ; c_{2}^{\prime}\right\rangle, \ldots,\left\langle p_{m}\left(\vec{x}_{m}^{\prime}\right) ; c_{m}^{\prime}\right\rangle \Rightarrow_{P}^{\star} \diamond$. Without loss of generality assume $F V(\mathrm{CLP}(w)) \cap F V\left(c_{i}^{\prime}\right)=\emptyset$ for all $i \in[1, m]$. Let $\vec{v}=\vec{x} \cdot \vec{x}_{1} \cdots \vec{x}_{l}$ and $\vec{v}^{\prime}=\vec{x}^{\prime} \cdot \vec{x}_{1}^{\prime} \cdots \vec{x}_{l}^{\prime}$. Let $g_{i}^{\prime} \in \mathcal{C}$ such that $\left\langle p_{i}\left(\vec{x}_{i}^{\prime}\right) ; 1\right\rangle \rightarrow_{P}^{\star}\left\langle\epsilon ; g_{i}^{\prime}\right\rangle$ and $c_{i+1}^{\prime}=c_{i}^{\prime} \otimes g_{i}^{\prime}$ for all $i \in[1, m)$. For all $i \in[1, m)$, put $g_{i}=\partial_{\vec{x}_{i}^{\prime}}^{\vec{x}_{i}}\left(g_{i}^{\prime}\right)$. Put $c_{1}=\partial_{\vec{v}^{\prime}}^{\vec{v}}\left(c_{1}^{\prime}\right)$ and for all $i \in[2, m]$, put $c_{i}=\partial_{\vec{x}_{i}^{\prime}}^{\vec{x}_{i}}\left(c_{i}^{\prime}\right)$. Then $c_{i+1}=c_{i} \otimes g_{i}$ for all $i \in[1, m)$. 
Let $\mathcal{O}^{\mathcal{C}}(P)=[F]_{\equiv}$. By proposition 4.1, $\left[p_{i}\left(\vec{x}_{i}\right):-g_{i}\right]_{\approx}=\left[p_{i}\left(\vec{x}_{i}^{\prime}\right):-g_{i}^{\prime}\right]_{\approx} \in F$ for all $i \in[1, m)$. By theorem 3.2, $\mathcal{O}^{\mathcal{C}}(P)=\mathcal{F}^{\mathcal{C}}(P)$ and by corollary 3.1, $\rho\left(\downarrow\left(\mathcal{F}^{\mathcal{C}}(P)\right)\right) \sqsubseteq$ $\mathcal{F}^{\operatorname{cod}(\rho)}(\rho(\downarrow(P)))$. Thus for $i \in[1, m)$ there exists $\left[p_{i}\left(\vec{x}_{i}\right):-f_{i}\right]_{\approx} \in F$ such that $\rho\left(\downarrow\left(g_{i}\right)\right) \subseteq f_{i}$. Put $f_{i}=0$ for all $i \in[m, l]$ to ensure $\left[p_{i}\left(\vec{x}_{i}\right):-f_{i}\right]_{\approx} \in F$ for all $i \in[m, l]$. Let $\left[p_{i}\left(\vec{x}_{i}\right):-d_{i}\right]_{\approx} \in D_{k}$ for all $i \in[1, l]$. Finally put $e_{n+1}=\mathcal{C}$, $e_{i}=d_{i} \cap\left(f_{i} \rightarrow^{\prime} e_{i+1}\right)$ for all $i \in[1, l]$ and $e_{0}=C \cap\left(\rho(\downarrow(c)) \rightarrow^{\prime} e_{1}\right)$. The inner induction is on $i$ and is used to show $\rho\left(\downarrow\left(c_{i}\right)\right) \subseteq e_{i}$ for all $i \in[1, m]$.

base case: Now $c_{1}=\partial_{\vec{v}^{\prime}} \overrightarrow{v_{1}}\left(c_{1}^{\prime}\right) \unlhd \partial_{\vec{y}}^{\vec{x}}\left(\bar{\exists}_{\vec{y}}\left(c^{\prime \prime}\right)\right) \in C$. Thus $\rho\left(\downarrow\left(c_{1}\right)\right) \subseteq C$. Furthermore, $c_{1}=\partial_{\vec{v}^{\prime}}^{\vec{v}}\left(c_{1}^{\prime}\right) \unlhd \partial_{\vec{v}^{\prime}}^{\vec{v}}\left(c^{\prime}\right)=c$. Thus $\rho\left(\downarrow\left(c_{1}\right)\right) \subseteq \rho(\downarrow(c))$. Moreover, $c_{1} \unlhd \partial_{\vec{y}}^{\vec{x}}\left(\bar{\exists}_{\vec{y}}\left(c^{\prime \prime}\right)\right) \in$ $\partial_{\vec{u}}^{\vec{x}}\left(\bar{\exists}_{\vec{y}}(e)\right) \subseteq \bar{\forall}_{\vec{x}}\left(e_{0}\right) \subseteq e_{0}$. Thus $\rho\left(\downarrow\left(c_{1}\right)\right) \subseteq e_{0}$. However, $e_{0}=C \cap\left(\rho(\downarrow(c)) \rightarrow{ }^{\prime} e_{1}\right)$. Thus $\rho\left(\downarrow\left(c_{1}\right)\right) \subseteq C \cap\left(\rho(\downarrow(c)) \rightarrow^{\prime} e_{1}\right)$ and $\rho\left(\downarrow\left(c_{1}\right)\right) \subseteq \rho\left(\downarrow\left(c_{1}\right)\right) \cap C \cap\left(\rho(\downarrow(c)) \rightarrow^{\prime} e_{1}\right)$ $=\rho\left(\downarrow\left(c_{1}\right)\right) \cap\left(\rho(\downarrow(c)) \rightarrow^{\prime} e_{1}\right)=\rho\left(\downarrow\left(c_{1}\right)\right) \cap \rho(\downarrow(c)) \cap\left(\rho(\downarrow(c)) \rightarrow^{\prime} e_{1}\right)=\rho\left(\downarrow\left(c_{1}\right)\right) \cap$ $\rho(\downarrow(c)) \cap e_{1}=\rho\left(\downarrow\left(c_{1}\right)\right) \cap e_{1}$. Therefore $\rho\left(\downarrow\left(c_{1}\right)\right) \subseteq e_{1}$ as required.

inductive case: Suppose $\rho\left(\downarrow\left(c_{i}\right)\right) \subseteq \varphi\left(e_{i}\right)$. Now $\rho\left(\downarrow\left(c_{i+1}\right)\right)=\rho\left(\downarrow\left(c_{i} \otimes g_{i}\right)\right)$ $\subseteq \rho\left(\downarrow\left(c_{i}\right)\right) \cap \rho\left(\downarrow\left(g_{i}\right)\right) \subseteq e_{i} \cap \rho\left(\downarrow\left(g_{i}\right)\right) \subseteq e_{i} \cap \rho\left(\downarrow\left(g_{i}\right)\right) \subseteq e_{i} \cap f_{i} \subseteq\left(f_{i} \rightarrow^{\prime} e_{i+1}\right) \cap f_{i}$ $=e_{i+1}$. Therefore $\rho\left(\downarrow\left(c_{i+1}\right)\right) \subseteq e_{i+1}$ as required.

Thus $\rho\left(\downarrow\left(c_{m}\right)\right) \subseteq e_{m} \subseteq d_{m}$ so that $c_{m} \in d_{m}$. Let $d_{m}^{\prime}=\partial_{\vec{x}_{m}}^{\vec{x}_{m}^{\prime}}\left(\bar{\exists}_{\vec{x}_{m}}\left(d_{m}\right)\right)$ and observe that $\left[p_{m}\left(\vec{x}_{m}^{\prime}\right):-d_{m}^{\prime}\right]_{\approx}=\left[p_{m}\left(\vec{x}_{m}\right):-d_{m}\right]_{\approx} \in D_{k}$. Put $c_{m}^{\prime \prime}=\partial_{\vec{x}_{m}^{\prime}}^{\vec{x}_{m}^{\prime}}\left(\bar{\exists}_{\vec{x}_{m}}\left(c_{m}\right)\right)$ so that $c_{m}^{\prime} \unlhd c_{m}^{\prime \prime} \in d_{m}^{\prime}$. By the inductive hypothesis $\left\langle p_{m}\left(\vec{x}_{m}^{\prime}\right) ; c_{m}^{\prime}\right\rangle \nRightarrow_{P}^{\star} \diamond$ which is a contradiction and hence $\bar{\exists}_{\vec{y}}\left(c^{\prime \prime}\right) \notin \bar{\exists}_{\vec{y}}(e)$ as required.

The result follows.

Proof for theorem 5.1

Let $\mathcal{D}^{\rho, \mathcal{C}}(P)=[D]_{\equiv},[p(\vec{y}):-e]_{\approx} \in D$ and $c^{\prime \prime} \in \bar{\exists}_{\vec{y}}(e)$. Thus $\bar{\exists}_{\vec{y}}\left(c^{\prime \prime}\right) \in \bar{\exists}_{\vec{y}}\left(\bar{\exists}_{\vec{y}}(e)\right)=$ $\bar{\exists}_{\vec{y}}(e)$. Suppose, for the sake of a contradiction, that $\left\langle p(\vec{y}) ; c^{\prime \prime} ; 1\right\rangle=s_{1} \Rightarrow_{P}^{\star} s_{n} \Rightarrow_{P} \diamond$ where $s_{i}=\left\langle g_{i} ; c_{i} ; h_{i}\right\rangle$. Let $k=\max \left(\left\{\max \left(h_{i}\right) \mid 1 \leq i \leq n\right\}\right)$. Suppose $\left(\mathcal{D}_{P}^{\rho, \mathcal{C}}\right)^{k}(\top)=$ $\left[D_{k}\right]_{\equiv}$. Since $D \sqsubseteq D_{k}$ and by lemma 5.1 there exists $\left[p(\vec{y}):-e^{\prime}\right] \approx \in D_{k}$ such that $\bar{\exists}_{\vec{y}}\left(c^{\prime \prime}\right) \notin \bar{\exists}_{\vec{y}}\left(e^{\prime}\right)$. Since $\bar{\exists}_{\vec{y}}(e) \subseteq \bar{\exists}_{\vec{y}}\left(e^{\prime}\right)$ it follows that $\bar{\exists}_{\vec{y}}\left(c^{\prime \prime}\right) \notin \bar{\exists}_{\vec{y}}(e)$ which is a contradiction. The result follows. 